\title{
A PROPÓSITO DE PIKETTY: EVOLUCIÓN DE LA DESIGUALDAD EN ESPAÑA
}

\section{ABOUT PIKETTY: EVOLUTION OF INEQUALITY IN SPAIN}

\author{
VÍCTOR LUQUE \\ Departamento de Economía Aplicada I \\ Universidad Complutense de Madrid \\ victorluquedeharo@gmail.com
}

Fecha recepción: Abril 2015

Fecha de aceptación: Junio 2015

\begin{abstract}
RESUMEN
Este trabajo estudia la evolución de la desigualdad en España, desde principios de la década de los ochenta, tanto en los ingresos como en la riqueza, atendiendo también a los cambios en la composición típica de ambas variables, en los diferentes cuantiles. Del análisis de las citadas magnitudes, se constata que determinadas figuras tributarias proyectan una desigual presión fiscal sobre los distintos segmentos de la población. Por otra parte, también se analizan los aspectos relativos a la difusión de los conocimientos, la inversión en educación, el crecimiento económico y el crecimiento demográfico, a los que Piketty califica como principales fuerzas de convergencia y divergencia sobre la distribución. Todo ello nos permite dilucidar el impacto que las citadas variables han tenido sobre la desigualdad y la forma en que las reformas institucionales implimentadas en estos campos han condicionado el desarrollo de las mencionadas fuerzas, así como presentar una previsión respecto a la forma e intensidad de su influencia futura en la evolución de la distribución.
\end{abstract}

PALABRAS CLAVE: Desigualdad, Piketty, Distribución de los ingresos, Distribución de la riqueza, Composición típica de los ingresos, Composición típica de la riqueza, España.

\section{ABSTRACT}

This paper studies the evolution of the inequality in Spain, both in terms of income and wealth, from the beginning of the eighties. Also attending to the changes in the typical composition of both variables in the different quantiles, during the period analyzed. From the analysis of these magnitudes, it appears that certain tax figures project an uneven tax burden on different segments of the population. Moreover, issues relating to the dissemination of knowledge, investment in education, economic growth and population growth, which Piketty qualifies as major forces of convergence and divergence on the distribution are also analyzed. This allows us to elucidate the impact of the mentioned variables have on inequality and how the implemented institutional reforms in these fields have conditioned the development of the mentioned forces and provide an estimate regarding the form and intensity of their future influence on the evolution of the distribution. 
Luque, Víctor. A propósito de Piketty: evolución de la desigualdad en España.

KEY WORDS: Inequality, Piketty, Incomes distribution, Wealth distribution, Incomes typical composition, Wealth typical composition, Spain.

JEL: D31, E62, J11, I28

\section{INTRODUCCIÓN}

La publicación de la obra de Piketty El Capital en el Siglo XXI sobre la evolución a largo plazo de la desigualdad, tanto en la riqueza como en los ingresos, ha arrojado luz sobre numerosas cuestiones en las que la falta de evidencia empírica, junto con la existencia de conflictos de intereses a flor de piel, dificultaba discutir de forma rigurosa sobre el tema ${ }^{1}$. Una de las claves de la gran aceptación de este estudio es que se realiza desde una perspectiva económica implícitamente bastante convencional, a pesar de que en numerosas ocasiones alude a factores políticos y a la existencia de conflictos de intereses ${ }^{2}$.

Ya en el nacimiento de la economía política en Francia y Reino Unido, la desigualdad ocupaba un lugar central en los análisis. Los economistas clásicos, consideraban la distribución de la producción entre las clases sociales como determinante del proceso de acumulación de capital. Para Malthus (1846), el diferente ritmo de crecimiento de la población y de la producción -debido principalmente a la escasez de tierras fértiles- era la principal amenaza. Ricardo $(1817)^{3}$, por su parte, temía el incremento del precio al arrendamiento de tierras, consecuencia de la fertilidad diferencial entre tierras y de la existencia de rendimientos decrecientes al aplicar más factor trabajo a una cantidad dada de tierra, como resultado del aumento de la población y las presiones por parte de la demanda, lo cual conllevaría a un incremento de la participación de los terratenientes en la renta nacional. En el prefacio de su obra más importante, "Principios de economía política y tributación" afirma que "el principal problema de la economía es determinar las leyes que regulan la distribución". Marx consideraba que el proceso de competencia entre capitales propio del funcionamiento del capitalismo conllevaba una tendencia a la centralización y concentración, lo cual conduce a una polarización de la situación entre propietarios de esos medios cada vez más concentrados y aquellos que tienen que vender su fuerza para sobrevivir. Así, este empeoramiento de las condiciones aparejado con el desarrollo tecnológico serían condiciones necesarias para la propia superación del capitalismo y el establecimiento de una sociedad comunista en la cual no existiese desigualdad.

Más tarde, autores como Kuznets ${ }^{4}$ pronosticaron una evolución más positiva de la desigualdad. Éste, consideraba que si bien en una primera fase del desarrollo económico la desigualdad tiende a aumentar, a lo largo del proceso de desarrollo a medida que el capital humano gana importancia sobre el capital físico la tendencia sería la contraria: la reducción de la desigualdad.

\footnotetext{
${ }^{1}$ Piketty (2014): ¿Un debate sin fuentes?: “Durante mucho tiempo los debates intelectuales y políticos sobre la distribución de la riqueza se alimentaron de muchos prejuicios y de muy pocos hechos.” Pág. 2.

${ }^{2}$ Vid. Piketty (2014): Págs. 20, 274, 314, 330.

${ }^{3}$ Ricardo, D. (1817): Principios de economía política y tributación.

${ }^{4}$ Kuznets, S. (1955): "Economic Growth and Income Inequality", The American Economic Review, American Economic Association, vol. 45, núm. 1, marzo de 1955, Págs. 1-28.
} 
Luque, Víctor. A propósito de Piketty: evolución de la desigualdad en España.

En las últimas décadas, muchos economistas del mainstream han tendido -la mayoría siguen haciéndolo ${ }^{5}$ - a caracterizar la desigualdad como una fuente de incentivos para el esfuerzo y la creatividad, de la cual la sociedad en su conjunto se beneficia y, a señalar que el establecimiento de altos tipos impositivos a los ricos y de ayudas a los pobres pueden perjudicar el crecimiento económico ${ }^{6}$. Premios nobel de economía como Robert Lucas han destacado el peligro que suponía centrarse en los temas de desigualdad para el correcto desempeño económico?.

Sin embargo, también existen numerosos economistas que alertan de los peligros y los costes que implica el aumento de la desigualdad: algunos estudios como el de Ostry et al. (2014) señalan que -siendo el resto de cosas igual- los países con altos niveles de desigualdad tienden a experimentar niveles de crecimiento menores y más volátiles, mientras que el crecimiento de aquellos con niveles más bajos de desigualdad tiende a ser superior y menos volátil. Wilkinson, Pikett y Chafe (2011) analizan los efectos de la desigualdad en variables sociales y sanitarias como la mortalidad infantil, la esperanza de vida, las tasas de criminalidad, la obesidad, las enfermedades mentales, etc. Llegan a la conclusión de que no solo los problemas sociales y sanitarios son más frecuentes entre la gente pobre que entre la gente rica sino que, además, el alcance de estos problemas es muy superior en las sociedades más desiguales. Otros autores como Martin Gilens destacan el peligro que supone para la democracia, señalando que los elevados niveles de desigualdad existentes en los EEUU han llevado a que "las preferencias de la mayoría de los ciudadanos americanos apenas tengan impacto en las políticas gubernamentales" ${ }^{8}$ así, cuando las preferencias de los ricos difieren de aquellas del público en general las políticas públicas reflejan las preferencias de los más ricos, excepto en excepcionales momentos de gran movilidad social. Lo cual puede ocasionar un círculo vicioso: los mayores niveles de desigualdad llevan a una elección de políticas públicas que favorecen a los más ricos lo cual favorece el aumento de la desigualdad ${ }^{9}$.

La evolución de la desigualdad a nivel internacional está aumentando o reduciéndose en función de si se analiza dentro de los países o entre ellos ${ }^{10}$. Glenn Firebaugh ${ }^{11}$ destacó que una de las tendencias principales de la desigualdad mundial daba muestras de estar invirtiéndose (pasando de la creciente desigualdad entre países y la constante o menguante desigualdad dentro de los países, a la desigualdad menguante entre países y la creciente desigualdad dentro de ellos). François Bourgiognon (2013) descubrió que, mientras la desigualdad planetaria (entre economías nacionales), medida en función de la renta media per cápita, sigue disminuyendo, la distancia entre los más ricos y los más pobres a escala global sigue creciendo, y los diferenciales de cada país continúan incrementándose.

\footnotetext{
${ }^{5}$ Por ejemplo Koo, R. C (2014) Pág. 98.

${ }^{6}$ Wade, R. H. (2014). The Piketty phenomenon and the future of inequality. real-world economics review, 2. Pág. 4.

7 "Of the tendencies that are harmful to sound economics, the most seductive, and in my opinion, the most poisonous, is to focus on questions of distribution” en Lucas, R. E. (2004) Pág. 20.

${ }^{8}$ Guiles, M (2012) Pág. 1.

${ }^{9}$ Bartels (2008)

${ }^{10}$ Piketty (2014): "Regardless of what measure is used, the world crearly seems to have entered a phase in which rich and poor countries are converging in income". Pág. 67.

${ }^{11}$ Firebaugh, G. (2009). The new geography of global income inequality. Harvard University Press.
} 
Luque, Víctor. A propósito de Piketty: evolución de la desigualdad en España.

La tesis central del libro es que una pequeña diferencia entre la tasa de retorno del capital ( $r$ ) y la tasa de crecimiento en la economía (g) puede, en el largo plazo, tener poderosos efectos desestabilizadores en la estructura y en las dinámicas de la desigualdad social ${ }^{12}$. Lo cual se da especialmente en regímenes de lento crecimiento. La riqueza acumulada es cada vez más importante y la heredada crece a un ritmo mayor que la producción y el ingreso. La combinación de estos factores con la existencia de rendimientos superiores del capital a medida que mayor es la riqueza inicial da como resultado un incremento en la concentración del capital.

Sin embargo, a lo largo del libro y en posteriores documentos aclaratorios ${ }^{13}$, Piketty explica que no hay que caer en determinismos económicos:

La historia de la distribución de la riqueza es siempre profundamente política y no podría resumirse en mecanismos puramente económicos (...) La historia de las desigualdades depende de las representaciones que se hacen los actores económicos, políticos y sociales, de lo que es justo y de lo que no lo es, de las relaciones de fuerza entre esos actores y de las elecciones colectivas que resultan de ello; es el producto conjunto de todos los actores interesados. (...) El resultado de esta historia dependerá de las representaciones que se hacen las diferentes sociedades de las desigualdades, y de las políticas e instituciones que se atribuyen para modelarlas y transformarlas, en uno $u$ otro sentido. ${ }^{14}$

En su obra, Piketty apunta a diferentes fuerzas que influyen en la evolución de la desigualdad. Las principales fuerzas divergentes -a parte de la mencionada $r>g-$ son la desaceleración del crecimiento demográfico y de la productividad, mientras que la principal fuerza de convergencia es el proceso de difusión de los conocimientos y de inversión en la capacitación y la formación de habilidades. Es importante señalar que la fuerza de divergencia $r>g$ no se debe a una imperfección de mercado, sino que al contrario, cuanto más perfectos ${ }^{15}$ sean los mercados financieros más fácil es que se cumpla $-y$ que la diferencia entre ambas variables sea mayor- esta desigualdad ${ }^{16}$.

Piketty destaca la igualdad contable $\alpha=r \times \beta$ como la primera ley fundamental del capitalismo. Donde:

- a representa el porcentaje que representan los beneficios en la renta nacional;

- $r$ es la rentabilidad media del conjunto de los capitales.

- $\quad \beta$ es la relación o ratio entre el capital y los ingresos nacionales.

Señala que debe ser considerada como tal puesto que permite vincular de manera simple y transparente los tres conceptos más importantes para el análisis del sistema capitalista: 1) La relación capital / ingreso; 2) La participación del capital en el ingreso y 3) La tasa de rendimiento del capital.

Advierte que esta identidad no nos indica cómo se determinan estas tres magnitudes y, en particular, no nos dice cómo se establece la relación capital/ingreso en un país, relación que mide en cierta manera la intensidad capitalística de una sociedad dada. A fin de avanzar en esta dirección, habremos de introducir otros mecanismos y otras

\footnotetext{
12 Vid Piketty (2014) Pág. 77.

${ }^{13}$ Piketty (2015) Pág. 69.

${ }^{14}$ Piketty (2014) Pág. 36.

${ }^{15}$ En el sentido del cumplimiento de los supuestos típicos de la economía neoclásica.

${ }^{16}$ Piketty (2014) Pág. 91.
} 
Luque, Víctor. A propósito de Piketty: evolución de la desigualdad en España.

nociones, en particular la tasa de ahorro y de inversión, así como la tasa de crecimiento.

Al introducir de los mecanismos comentados en el párrafo anterior, Piketty los relaciona de manera que introduce la que para él "puede ser considerada como la segunda ley fundamental del capitalismo: $\beta=s / g$ ". Donde $s$ representa la tasa de ahorro del país considerado mientras que $g$ es la tasa de crecimiento de su ingreso nacional. En la explicación del funcionamiento de esta ley aclara que no permite explicar los shocks de corto plazo a los que el ratio capital ingresos esta sujeto pero que, sin embargo, nos permite entender el nivel de equilibrio potencial al que esta ratio tiende en el largo plazo, cuando los efectos de los shocks y las crisis de corto plazo se hayan disipado ${ }^{17}$.

El autor se cuestiona ¿Por qué la concentración en los ingresos y en la riqueza se incrementa a medida qué estamos en la parte más alta de la escala de distribución? Una de las razones que ofrece es que la tasa de retorno sobre los capitales se incrementa a medida que aumenta la riqueza. Otra es la herencia, en la medida en que las fortunas pasan de una generación a otra y que cuando tales patrimonios alcanzan un volumen suficientemente grande, gracias a los rendimientos superiores al crecimiento de la economía, permiten una vida cómoda a su propietario de forma simultánea al aumento de su tamaño ${ }^{18}$.

En la contabilización de la riqueza y el capital -términos que a lo largo del libro se usan indistintamente- Piketty incluye todos aquellos activos que pueden ser poseídos e intercambiados en el mercado ${ }^{19}$. No obstante, esta metodología de contabilización ha recibido numerosas críticas doctrinales. Varoufakis (2014), por ejemplo, muestra su desacuerdo con la asimilación entre riqueza y bienes de capital y entre rentabilidad del capital y rendimientos por unidad de riqueza ${ }^{20}$. Rallo, J. R (2015), por su parte, considera que la inclusión del capital humano en la citada contabilización implicaría unos resultados con unos niveles de desigualdad en la riqueza y el capital mucho menores a los mostrados ${ }^{21}$. Ghosh, J. (2014) critica la

\footnotetext{
${ }^{17}$ Vid. Piketty (2014) Pág. 168

${ }^{18}$ Bauman (2014) comparte la esencia de esta argumentación cuando afirma que "Los ricos se están enriqueciendo sólo porque son ricos. Los pobres se empobrecen solo porque son pobres. Hoy en día, la desigualdad se agrava siguiendo su propia lógica y su propio ritmo. No necesita ninguna otra ayuda, estímulo, presión o impuesto externo." Pág. 22.

${ }^{19}$ En el marco de este libro, el capital se define como "el conjunto de los activos no humanos que pueden ser poseídos e intercambiados en un mercado. El capital incluye sobre todo el conjunto del capital inmobiliario (inmuebles, casas) utilizado como vivienda, y el capital financiero y profesional (edificios, equipos, máquinas, patentes, etc.) utilizado por las empresas y las agencias gubernamentales." Por lo tanto, esto incluye "todas las formas de riqueza que, a priori, pueden ser poseídas por individuos (o grupos de individuos) y transmitidas o intercambiadas en un mercado de modo permanente (...) El capital no es un concepto inmutable: refleja el estado del desarrollo y las relaciones sociales que rigen a una sociedad dada." Vid. Pág. 46.

${ }^{20}$ En este artículo, Varoufakis reconoce la utilidad de la medición de la riqueza en un mundo en el que la riqueza relativa determina el poder relativo entre aquellos que tienen montones de riqueza y aquellos que apenas tienen nada. Sin embargo, señala que el crecimiento de una economía no se basa en la riqueza sino en un tipo particular de ésta -los bienes de capital-. Por tanto, según Varoufakis, la definición de capital que usa Piketty es incapaz de ayudarnos a comprender el vínculo que existe entre capital y PIB, o en el incremento en el stock de capital -bien sea por precio o por cantidad-; una relación que es esencial para la narrativa del profesor Piketty.

${ }^{21}$ Artículo en su blog http://juanramonrallo.com/2015/01/los-tres-errores-clave-de-thomas-piketty/. La principal justificación que da Piketty para la no inclusión del capital humano en la contabilización del capital es que "este capital no puede ser poseído por otra persona ni intercambiado en un mercado, o por lo menos no de modo
} 
Luque, Víctor. A propósito de Piketty: evolución de la desigualdad en España.

incongruencia entre la contabilización del capital como la suma de los valores de mercado -puesto que en última instancia es reflejo de relaciones sociales- con la idea de la rentabilidad del capital entendida como productividad marginal de éste, la cual es implícitamente una de las bases de su formulación teórica ${ }^{22}$. Una de las principales ventajas que tiene la definición de capital utilizada es que permite la homogeneización de los diferentes tipos de riqueza a sus precios de mercado, posibilitando la comparación en diferentes momentos y países. Otras ventajas destacables son que: permite capturar gastos o inversiones realizados en activos que no son medidos como parte de la inversión en las cuentas nacionales; en comparación con el método alternativo de acumulación de flujos de inversión pasados, incorpora los efectos de los cambios tecnológicos, los cambios en precios $\mathrm{u}$ otras perturbaciones que pudieran afectar a la productividad del capital23.

Los análisis que se realizan de la distribución y de su evolución en diferentes países muestran que -pese a existir en las últimas décadas una tendencia a la concentración de la riqueza y los ingresos en la práctica totalidad- las evoluciones difieren notablemente. Los países del Norte de Europa y Japón experimentaron un incremento de la concentración tanto en el conjunto de rentas como en la riqueza, aunque especialmente en la primera, en los cuantiles superiores mucho menor que EEUU, Reino Unido y otros países anglosajones. Estas diferentes evoluciones nos muestran, en primer lugar que, pese a que pueda existir una tendencia en el funcionamiento normal del capitalismo al aumento de la concentración, no es algo que esté totalmente determinado sino que diferentes factores como el tipo de políticas implementadas o los marcos institucionales tienen una capacidad de atenuar esta tendencia incluso revertirla en un grado mayor del que se puede deducir de alguno de los capítulos del libro.

En segundo lugar, ponen de manifiesto que economías con un menor grado de desigualdad no son necesariamente menos dinámicas y que economías con mayor desigualdad no necesariamente -más bien al contrario- tienen un desempeño mejor en la mejora de las condiciones materiales de vida de la mayoría de la población ${ }^{24}$.

Expuesto lo anterior, este trabajo intenta dar respuesta a las siguientes cuestiones:

- ¿Cuál es la situación de la desigualdad en España?

- ¿Cómo ha sido su evolución reciente?

- ¿En que medida las modificaciones institucionales que se han venido realizando en los últimos años afectan a las fuerzas, tanto convergentes como divergentes, que señala Piketty?

Para ello se estructura en cuatro apartados. El primero está referido al análisis de la desigualdad de la renta en España. El segundo, analiza el grado de concentración de la riqueza. En el tercer apartado se tratan los factores que, según Piketty, actúan

permanente. Esto constituye una diferencia esencial respecto de las demás formas de capital” Piketty (2014) Pág. 60 .

${ }^{22}$ Vid. Jayati Ghosh, "Piketty and the resurgence of patrimonial capitalism". real-world economics review, issue no. 69, 7 Oct 2014, Pág. 141.

${ }^{23}$ Weil, D. N. (2015) Pág. 2.

${ }^{24}$ Wade, R. H. (2014). The Piketty phenomenon and the future of inequality. Real-world economics review, 2. Pág. 4. 
Luque, Víctor. A propósito de Piketty: evolución de la desigualdad en España.

como fuerzas convergentes y divergentes de la concentración de los ingresos y la riqueza. Finalmente se presentan unas conclusiones.

\section{ANÁLISIS DE LA SITUACIÓN DE LA DESIGUALDAD DE LA RENTA EN ESPAÑA}

A pesar de que una de las principales razones del éxito del análisis de Piketty sobre la evolución de la desigualdad ha sido el prolongado período temporal para el que proporciona datos sobre la desigualdad, para el caso español las limitaciones en este aspecto son importantes. Esta es la causa principal de que el periodo temporal del análisis que se hace a continuación abarque solo desde 1981 hasta 2012. Esta circunstancia implicará que algunas de las fuerzas o leyes que se describieron anteriormente no actúen de forma plena, como por ejemplo la segunda ley fundamental ( $\beta=\mathrm{s} / \mathrm{g}$ ), puesto que su potencial explicativo es en el largo plazo. Además, la otra condición que Piketty establece para el cumplimiento de esta ley es que a largo plazo los precios de los activos evolucionen en promedio de la misma forma que los precios al consumidor. Si bien es discutible que esta condición se de en el largo plazo ${ }^{25}$, es evidente que en España y en el periodo analizado esto no ha sido así26 por lo que consideraremos que en lugar de una ley nos encontramos con dos potentes elementos condicionantes de la relación riqueza / renta.

Tanto en el análisis que se hace a continuación, sobre la desigualdad en el conjunto de rentas, como en el siguiente, sobre la desigualdad de la riqueza, se realizan principalmente mediante el estudio de la concentración de ambas variables en distintos cuantiles. Las ventajas de la utilización de cuantiles (ya sean deciles, percentiles, etc.) se derivan de las facilidades a la hora de comparar entre distintos países y periodos temporales. Además, constituye un indicador pertinente para valorar la desigualdad de una sociedad, ya que toma en cuenta no sólo la existencia de ingresos o patrimonios extremos, sino también el número de personas beneficiadas por esos valores tan elevados ${ }^{27}$.

Creo que es necesario destacar que, aunque pueda parecer una obviedad, las jerarquías operantes no son exactamente las mismas para los ingresos del trabajo que para la riqueza, por lo que no encontraremos exactamente los mismos individuos dentro de cuantiles similares. Sin embargo, en las sociedades modernas existe una correlación positiva, aunque no perfecta, entre ambas variables ${ }^{28}$. Siendo, por tanto, la desigualdad social multidimensional, al igual que el conflicto político 29 . Esta multidimensionalidad es uno de los motivos de que tanto los análisis de Piketty como la base de datos "The New Income Data Base" se realicen principalmente mediante análisis por cuantiles en lugar de hacerlo mediante índices sintéticos como por ejemplo el índice de Gini. Puesto que:

\footnotetext{
${ }^{25}$ Vid. Weil, D. N. (2015): Pág. 6.

${ }^{26}$ Más adelante se recogen algunos datos que avalan esta afirmación como los relativos a la evolución de los activos inmobiliarios y los bursátiles.

${ }^{27}$ Piketty (2014): Pág. 277.

${ }^{28}$ Además, según exponen Alvaredo, Atkinson, Piketty y Saez, esta correlación ha aumentado en EEUU durante las últimas décadas, lo cual nos hace suponer que en España se ha podido dar un movimiento en la misma dirección. Vid. Alvaredo, F., Atkinson, A. B., Piketty, T., \& Saez, E. (2013): Pág. 7.

${ }^{29}$ Ibíd. 278.
} 
Luque, Víctor. A propósito de Piketty: evolución de la desigualdad en España.

Estos coeficientes (...) pretenden resumir en un único indicador numérico la desigualdad completa de la distribución (...), lo que es muy simple y seductor a primera vista, pero inevitablemente un poco ilusorio. (...) es imposible resumir una realidad multidimensional mediante un indicador unidimensional, salvo si se simplifica en exceso esta realidad y se mezclan aspectos incomparables ${ }^{30}$.

Como se comentó anteriormente, este apartado se centrará en analizar la evolución de la concentración de los ingresos en España en las últimas décadas. En la tabla 1.1 se resume la participación en los ingresos totales que tiene cada uno de los segmentos analizados del decil con más ingresos. Por tanto, se incluyen tanto los ingresos procedentes del trabajo como aquellos procedentes de la propiedad del capital. Es importante el estudio dentro del primer decil y no de éste a nivel agregado puesto que como afirma Bauman (2014) para comprender la mutación que está actualmente en marcha, que es distinta de una mera fase de ciclo, tenemos que centrarnos en el $1 \%$ más rico, quizás incluso en el $0,1 \%$ más rico. Si no lo hacemos, no conseguiremos apreciar el verdadero impacto del cambio que se está produciendo, y que consiste en la degradación de la clase media al nivel del precariado. Sin embargo, sí que habría sido interesante poder contar también con datos sobre los ingresos de los otros deciles para ver la evolución que han seguido y hacer así un análisis más rico sobre el progreso de esta desigualdad, pero en la base de datos que se ha utilizado no están aún disponibles y la utilización de otras fuentes con diferente metodología habría reducido la homogeneidad de los datos.

Al estudiar la composición media de los ingresos, éstos se desagregan en ingresos salariales, ingresos empresariales, ingresos del capital y aquellos derivados de la revalorización del capital. Los ingresos salariales incluyen (salarios, pensiones y otros ingresos derivados del trabajo). Los ingresos empresariales incluyen los ingresos del trabajo por cuenta propia, los ingresos de las explotaciones agrarias y los ingresos de los pequeños negocios. Los ingresos del capital están formados por dividendos, intereses, rentas e ingresos de inversiones extranjeras y otras inversiones. Las ganancias de capital son las revalorizaciones en el precio de los distintos tipos de capital y riqueza ${ }^{31}$.

Las causas que explican la evolución de los ingresos son distintas para cada tipo de ingresos. En el caso de los ingresos del trabajo, los principales mecanismos que actúan son: 1) La oferta y demanda de habilidades; 2) El estado del sistema educativo; 3) El conjunto de reglas e instituciones que afectan en el funcionamiento del mercado de trabajo y en la determinación de los salarios. En el caso de los ingresos del capital, los principales factores de los que depende su evolución son: 1) Los comportamientos de ahorro e inversión; 2) Las normativas que regulan las donaciones y las sucesiones; 3) El funcionamiento del mercado inmobiliario y los mercados financieros ${ }^{32}$.

Para poner en contexto estas cifras puede ser útil señalar que en las sociedades en las que la desigualdad total en los ingresos del trabajo es relativamente baja (como

\footnotetext{
${ }^{30}$ Ibíd. Pág. 291

${ }^{31}$ Para más información sobre aspectos metodológicos vid. Notas metodológicas de Facundo Alvaredo y Luis Estévez. Disponibles en:

http://topincomes.parisschoolofeconomics.eu/TopIncomes/service/DownloadPdfServlet?fileName=Spain_Updat e2014_Sept_2.pdf Recuperado el 21 de mayo de 2015.

${ }^{32}$ Piketty (2014) Pág. 243.
}

Papeles de Europa 
Luque, Víctor. A propósito de Piketty: evolución de la desigualdad en España.

en los países escandinavos en los años de 1970-1980), el 10\% de los mejor pagados poseen alrededor del $20 \%$ del ingreso total. ${ }^{33}$

Tabla 1.1 Participación en los ingresos totales (incluidas las ganancias de capital).

\begin{tabular}{|c|c|c|c|c|c|c|}
\hline & Top 10-5\% & Top 5-1\% & Top 1-.5\% & Top .5-.1\% & Top.1-.01\% & Top.01\% \\
\hline 1981 & 11,46 & 13,62 & 2,65 & 3,04 & 1,39 & 0,55 \\
\hline 1989 & 12,08 & 15,02 & 2,99 & 3,49 & 1,72 & 0,82 \\
\hline 1995 & 11,61 & 13,94 & 2,85 & 3,30 & 1,55 & 0,59 \\
\hline 2006 & 10,57 & 13,51 & 3,07 & 4,32 & 3,07 & 2,27 \\
\hline 2012 & 10,63 & 12,77 & 2,65 & 3,16 & 1,76 & 1,00 \\
\hline
\end{tabular}

Fuente: Elaboración propia a partir de los datos de "The World Top Incomes Data Base".

Datos en términos porcentuales.

Si observamos la evolución de la participación de los ingresos de los segmentos más ricos de la población en los ingresos totales, vemos que el comportamiento de los diferentes segmentos no ha sido homogéneo ni entre ellos ni en el tiempo. En este sentido se diferencian cuatro subperíodos:

- El primer subperíodo (1981-1989) se caracteriza por incluir años de fuerte crecimiento, especialmente desde 1987 hasta 1989, con las tasas de crecimiento del PIB superiores al $4 \%$. Durante este subperíodo se produce una concentración de los ingresos en todos los segmentos pertenecientes al primer decil.

- El segundo subperíodo, que abarca desde 1990 a 1995, comienza con una desaceleración del crecimiento llegando incluso a tasas negativas durante el 1993. Año a partir del cual vuelve a repuntar el ritmo de crecimiento. Durante estos años las ganancias de capital son menores, siendo ésta una de las causas que explica que durante este subperíodo se produzca una caída en la participación en los ingresos de los segmentos analizados del primer decil.

- El tercer subperíodo (1996-2006) se caracteriza por ser una época de fuerte crecimiento y notables ganancias de capital. Es un período en el cual se produce un comportamiento diferencial entre los distintos segmentos. Mientras que aquellos que se sitúan entre el $10 \%$ y el $0,5 \%$ con más ingresos pierden participación en el total, aquellos que se sitúan a partir del 0,5\% mejoran aún más su participación, especialmente el grupo del 0,01\% más rico (que pasa de $0,59 \%$ a $2,27 \%)$.

- El cuarto subperíodo se inicia con la desaceleración del crecimiento a partir de 2007 y continúa con una contracción muy fuerte de la economía en forma de doble U, volviendo en el año 2012 (el último del que se disponen datos) a experimentar tasas negativas de crecimiento. Durante este subperíodo se reduce parcialmente la concentración de los ingresos que se había dado en el subperíodo anterior. Las caídas en la participación más fuertes se dan en los segmentos $(0,1 \%$ - 0,01\% y Top $0,01 \%)$ que más habían aumentado su participación en el período anterior (pasando de 3,07 \% de participación a 1,76 \% y de $2,27 \%$ a $1 \%$ respectivamente).

\footnotetext{
${ }^{33}$ Piketty (2014) Pág. 237.
} 
Luque, Víctor. A propósito de Piketty: evolución de la desigualdad en España.

En las últimas décadas ha existido una tendencia en España a una concentración de los ingresos en los percentiles más ricos. En el gráfico 1.1 podemos observar la diferente evolución que se produce en la participación en los ingresos del top 0,1\% si incluimos las ganancias de capital o si las excluimos. Si bien, como era de esperar ambas funciones presentan una clara correlación, las variaciones que se producen en la participación cuando incluimos las ganancias de capital son notablemente superiores a cuando están excluidas. La diferencia entre ambas funciones viene explicada simplemente por las ganancias de capital, es decir, por la revalorización del conjunto de activos propiedad de este segmento de la población. Esta diferencia es sustancialmente superior en los años de fuerte expansión económica, siendo el año 2006 en el que la diferencia entre ambas participaciones es mayor (incluyendo las ganancias de capital la participación alcanza el 5,34 \% en los ingresos totales mientras que cuando no se incluyen la participación es del 2,84\%). Esto viene explicado tanto por la revalorización de los activos inmobiliarios y de los activos bursátiles que se suele experimentar en las épocas de boom económico ${ }^{34}$ como por la alta concentración de la riqueza en un porcentaje reducido de la población, principalmente la financiera como veremos en el apartado 2.

Gráfico 1.1. Participación de los ingresos del $0,1 \%$ más rico en los ingresos totales

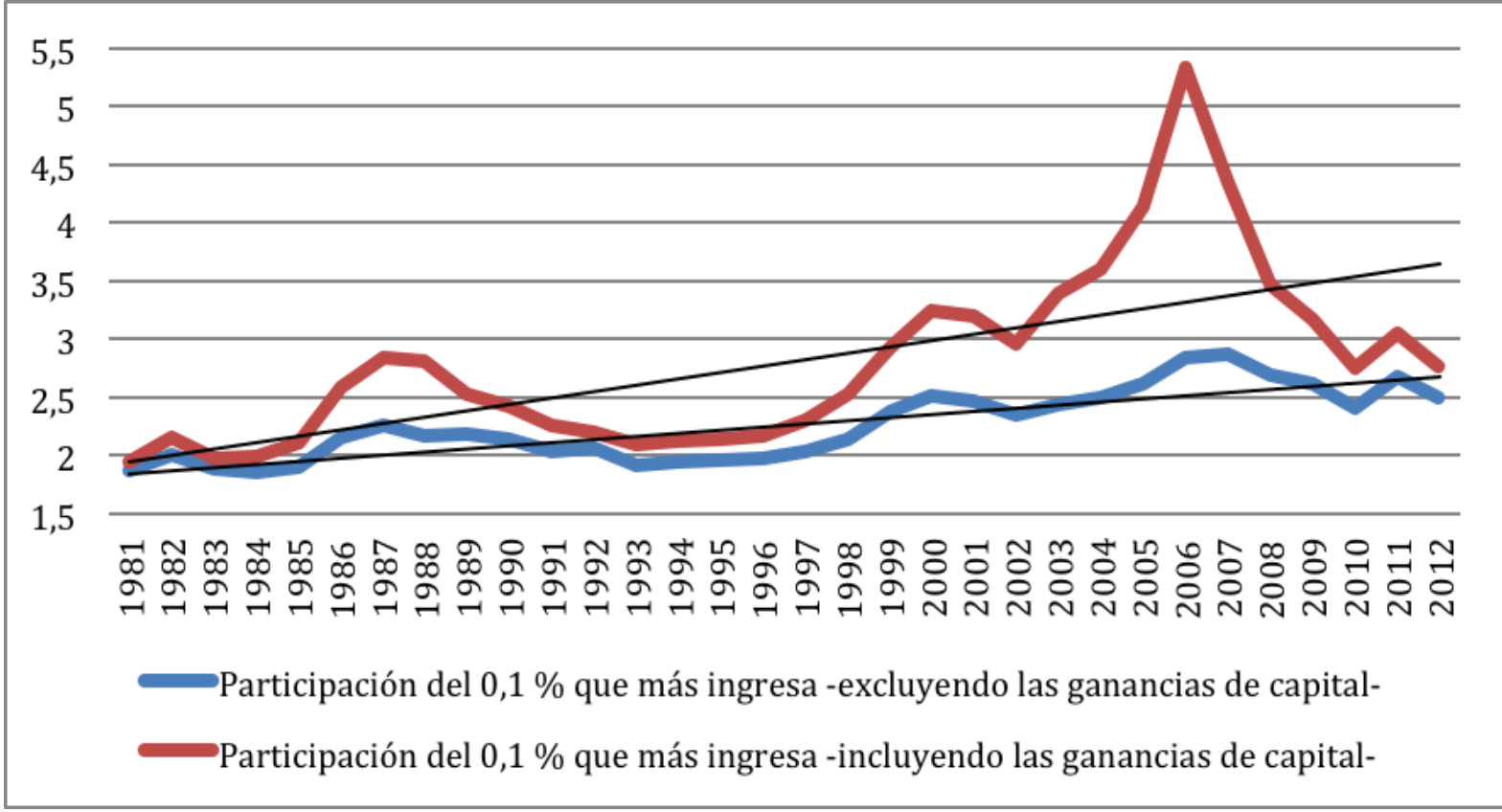

Fuente: Elaboración propia a partir de los datos de "The World Top Incomes Database". Participación en términos porcentuales.

La evolución de los diferentes componentes del ingreso del 0,1\% a lo largo de este período tiene notables diferencias: las ganancias por revalorización del capital y los ingresos salariales han sido los que han crecido a un ritmo más alto. Los dos componentes restantes, ingresos empresariales e ingresos del capital, han permanecido relativamente constantes. Ahora bien, mientras que el crecimiento de las ganancias de capital tiene una marcada estacionalidad, el incremento en los ingresos salariales se ha producido de forma paulatina a lo largo de todo el

\footnotetext{
${ }^{34}$ Desde 2002 a 2007 el valor nominal medio del m2 de suelo en España experimentó un crecimiento medio anual del 12,24 \% según la Sociedad de Tasación, la revalorización del índice IBEX-35 fue de 10,37 \% medio anual y el crecimiento del PIB nominal del 7,52\% anual.
} 
Luque, Víctor. A propósito de Piketty: evolución de la desigualdad en España.

período ${ }^{35}$. De esta forma, tanto en el gráfico anterior como en la Tabla 1.1 parece que se cumple la constatación expresada por Piketty según la cual la desigualdad tiende a evolucionar de forma procíclica. En efecto, se observa que en los momentos de mayor crecimiento económico, la participación de los beneficios en el ingreso nacional tiende a incrementarse, y los salarios más elevados generalmente se incrementan más que los salarios medios. Al contrario sucede en las épocas de recesión, varios factores no económicos, especialmente políticos, hacen que esos movimientos no dependan únicamente del ciclo económico ${ }^{36}$. En cualquier caso, en el medio largo plazo, se observa una tendencia a la concentración de los ingresos en ausencia de un marco institucional que lo corrija. Esta tendencia es contraria a la teorizada por Kuznets (1955) según la cual pese a que en un primer estadío del desarrollo se produce un aumento de la desigualdad, en una segunda fase la desigualdad se reduce progresivamente a medida que el propio desarrollo tecnológico hace que el capital humano vaya ganando importancia sobre el capital físico.

Respecto a la composición de los ingresos, se observa que la proporción de los provenientes de la propiedad del capital se incrementa significativamente en los sujetos que se encuentran más arriba en la distribución (Tabla 1.2). De esta forma, puede verse como los ingresos procedentes de los salarios y las pensiones van perdiendo peso, mientras que los procedentes de la actividad empresarial, los ingresos del capital y aquellos derivados de la revalorización del capital ganan importancia. Si atendemos a la composición de los ingresos de los individuos que se sitúan en la franja del 10 al $5 \%$ con mayores ingresos advertimos como, pese a ser parte del primer decir, el porcentaje de los ingresos que corresponden al grupo de salarios supera el $88 \%$ del total.

De hecho, cuando observamos la composición de los ingresos del 0,01\% más rico vemos como los salarios y las pensiones dejan de ser la fuente principal de ingresos y su lugar lo ocupan aquellos derivados de la revalorización del capital. Esta situación viene explicada tanto por el mayor peso de los capitales en la composición de su riqueza en comparación con el resto de individuos ${ }^{37}$, como por la mayor rentabilidad media ${ }^{38}$ que suelen obtener. Piketty explica esto último con dos argumentos: 1) por la existencia de economías de escala en la gestión de la cartera de inversión y 2) porque es más fácil para un inversor tomar riesgos y ser paciente si tiene reservas sustanciales ${ }^{39}$. A estas razones se podrían añadir otras como la existencia de determinadas imperfecciones en los mercados financieros ${ }^{40}$ y las

\footnotetext{
${ }^{35}$ Alvaredo (2009) Pág. 1155.

${ }^{36}$ Piketty (2014) Pág. 288.

${ }^{37}$ Pese a que no tienen que coincidir los individuos con mayores ingresos de aquellos con mayor riqueza sí que existe, en las sociedades modernas, una correlación postivia entre la posición relativa en la distribución de una de estas variables con la otra. En la mayoría de las sociedades Europeas y en EEUU la mitad de la población no posee virtualmente nada: el $50 \%$ más pobre posee menos del 10\% de la riqueza nacional, generalmente menos del $5 \%$. Piketty (2014): pp. 257. Según las estadísticas de Alvaredo, F., \& Saez, E. (2009), en el año 2007 en España más del $25 \%$ de la riqueza financiera era propiedad del $1 \%$ más rico.

${ }^{38}$ Este aspecto es duramente criticado por Rallo, J. R. Al señalar que los rendimientos superiores que Piketty destaca de los más ricos no son tales. Decir que la riqueza del $1 \%$ ha aumentado a un ritmo notablemente superior al del resto de la población no implica que se hayan mantenido dentro de cada cuantil los mismos individuos debido a la existencia de convergencia beta (movimiento de los individuos dentro de la distribución). http://juanramonrallo.com/2014/04/que-paso-con-los-superricos-de-1987/

${ }^{39}$ Piketty (2014:430)

${ }^{40}$ Stiglitz (2014) Pág. 161
}

Papeles de Europa 
rentas de monopolio ${ }^{41}$. A este respecto, es significativo que en todos los países para los que se disponen de este tipo de datos y en todos los períodos el porcentaje de ingresos derivados del trabajo disminuye rápidamente conforme nos desplazamos a la parte superior del primer decil, y la participación de los ingresos derivados del capital aumenta de forma pronunciada ${ }^{42}$. Como consecuencia de todo lo anterior, la desigualdad en los ingresos del capital suele ser superior incluso que la desigualdad en la propiedad del mismo ${ }^{43}$.

A partir de estos datos es fácil presumir a quién benefician regulaciones fiscales como el diferente tratamiento a las rentas y las ganancias del capital respecto a las rentas del trabajo, en beneficio de las primeras ${ }^{44}$ o a quién beneficia la existencia de instrumentos como las SICAV para la gestión de patrimonios ${ }^{45}$.

Tabla 1.2. Composición media de los ingresos ${ }^{46}$ del primer decil durante el período 1981-2012

\begin{tabular}{|c|c|c|c|c|}
\hline & Salarios & Empresariales & Capital & $\begin{array}{c}\text { Ganancias de } \\
\text { capital }\end{array}$ \\
\hline Top $10-5 \%$ & 88,61 & 7,26 & 5,88 & 1,47 \\
\hline Top 5-1\% & 82,66 & 9,72 & 7,77 & 3,08 \\
\hline Top 1-0.5\% & 72,21 & 14,45 & 10,68 & 5,88 \\
\hline Top 0.5-0.1\% & 60,57 & 18,60 & 14,50 & 9,56 \\
\hline Top 0.1-0.01\% & 39,61 & 24,40 & 20,44 & 18,77 \\
\hline Top 0.01\% & 24,24 & 23,29 & 23,05 & 32,64 \\
\hline
\end{tabular}

Fuente: Elaboración propia a partir de los datos de "The World Top Incomes Database". Datos en términos porcentuales.

Se debe tener en cuenta, además, que los ingresos procedentes del capital se encuentran subestimados, debido tanto a la evasión fiscal como a la existencia de diferentes exenciones fiscales ${ }^{47}$. Por tanto, la concentración es presumiblemente mayor de la que reflejan los datos anteriormente expuestos. Un reciente estudio de Gabriel Zucman sugiere que el porcentaje de riqueza europea oculta en paraísos fiscales es superior al de EEUU. Según él, ello se debe al mayor incentivo que existe en Europa a la ocultación de capitales por los mayores niveles de gravamen ${ }^{48}$. Otra causa puede encontrarse en la menor coordinación de las agencias tributarias y en

\footnotetext{
${ }^{41}$ Ibíd. Pág. 90

42 Ibíd. Pág. 279

${ }^{43}$ Ibíd. Pág. 243

${ }^{44}$ Como ocurre actualmente en el IRPF. Véase Ley 35/2006, de 28 de noviembre, del Impuesto sobre la Renta de las Personas Físicas y de modificación parcial de las leyes de los Impuestos sobre Sociedades, sobre la Renta de no Residentes y sobre el Patrimonio.Vid. Oxfam, I. (2012) Pág. 31.

${ }^{45}$ Luque, M. A y Luque, V.(2014): “A Vueltas Con Las Sicav. Reflexiones Críticas Desde El Punto De Vista Económico-Tributario" en Revista Papeles de Europa, vol.27. no 2.

${ }^{46}$ Los ingresos salariales incluyen (salarios, pensiones y otros ingresos derivados del trabajo). Los ingresos empresariales incluyen los ingresos del trabajo por cuenta propia. Los ingresos de las explotaciones agrarias y los ingresos de los pequeños negocios. Los ingresos del capital están formados por dividendos, intereses, rentas e ingresos de inversiones extranjeras y otras inversiones. Las ganancias de capital son las revalorizaciones en el precio de los distintos tipos de capital y riqueza.

${ }^{47}$ Piketty (2014) Pág. 281 y ss.

${ }^{48}$ Zucman, G. (2013)
}

Papeles de Europa 
Luque, Víctor. A propósito de Piketty: evolución de la desigualdad en España.

la ausencia de transmisión automática de información bancaria entre los diferentes países que forman la Unión Europea a la que alude Piketty ${ }^{49}$.

\section{ANÁLISIS DE LA CONCENTRACIÓN DE LA RIQUEZA EN ESPAÑA}

A continuación pasamos a estudiar la evolución de la distribución y de la composición de la riqueza. Como ya se comentó anteriormente, en la práctica, la distribución de la propiedad del capital y de los ingresos resultantes está mucho más concentrada que la de los ingresos derivados del trabajo. De hecho, en la mayoría de los países, la riqueza se encuentra tan sumamente concentrada que un gran segmento de la población no es ni siquiera consciente de su existencia ${ }^{50}$.

En su libro, Piketty muestra la gran transformación que se produce en la naturaleza y composición del capital desde el siglo XVIII hasta la actualidad. Durante este período, la tierra agrícola deja de ser el principal componente de la riqueza total siendo sustituida por activos inmobiliarios, capital industrial, comercial y capital financiero ${ }^{51}$.

Grafico 2.1: Evolución de la riqueza neta por adulto y de su composición (1982-2007) Valores constantes a precios de 2010.

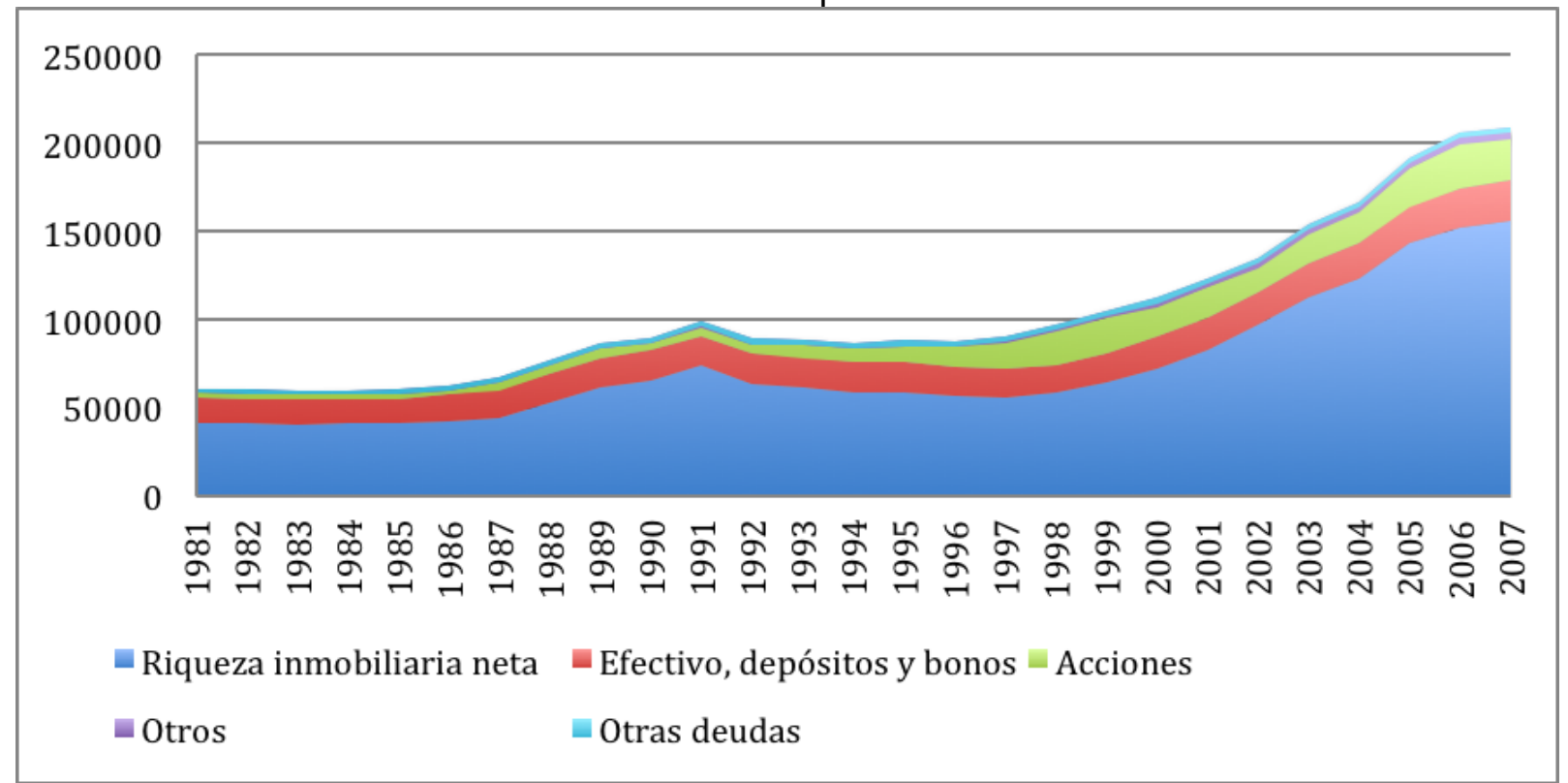

Fuente: Elaboración propia a partir de los datos de Income and Wealth Concentration in Spain from a Historical and Fiscal Perspective (tables and figures)

Desafortunadamente, para España -al contrario que para otros países como EEUU, Francia o Reino Unido- no se disponen de datos tan a largo plazo ${ }^{52}$. Esta circunstancia, al igual que se comentó en el apartado anterior referente a la concentración de las rentas, limitará el impacto de la segunda ley de Piketty y de otras fuerzas convergentes y divergentes que actúan principalmente en el largo plazo. Los elementos más destacables de la evolución de la riqueza en España

\footnotetext{
${ }^{49}$ Piketty (2014) Pág. 521 y ss.

${ }^{50}$ Piketty (2014) Pág. 259.

${ }^{51}$ En el capítulo 2 del libro podemos ver la evolución de la composición y la cuantía del capital medido en años de ingresos nacional en Inglaterra, Francia, Alemania, EEUU y Canadá.

${ }^{52}$ La Base de datos de Alvaredo para España estudia el período 1981-2007.
} 
Luque, Víctor. A propósito de Piketty: evolución de la desigualdad en España.

durante el período 1981-2007 son los siguientes: 1) La riqueza se ha incrementado a un ritmo alto durante el período (4,98\% de variación media anual). 2) Los activos inmobiliarios representan la mayor parte de la riqueza total (superior al $70 \%$ en todos los años analizados). 3) Consecuentemente, el crecimiento de la riqueza media viene explicado principalmente por el incremento de los precios y del número de los activos inmobiliarios (5,28 \% de tasa de variación media anual), así como por el aumento del valor de las acciones empresariales $(8,15 \%$ de tasa media de variación anual). Por el contrario, el crecimiento de la riqueza en forma de efectivo, depósitos y bonos ha sido notablemente inferior (1,85 \% de tasa de variación media anual).

Atendiendo a la evolución de la concentración de la riqueza en los segmentos más ricos de la población (gráfico 2.2), observamos que los individuos que se encuentran entre el $1 \%$ y el $0.1 \%$ han ganado participación en el total (pasando del 10,95 en 1982 al 14,07 \% en 2007). Sin embargo, durante ese mismo período, el porcentaje de riqueza correspondiente al top $0.1 \%$ se ha visto reducido considerablemente (del 7,48 al 5,58 \%). Esto se debe, principalmente, al hecho de que la riqueza inmobiliaria esta menos concentrada que la riqueza financiera ${ }^{53}$. A lo que habría que añadir el aumento de los precios de la propiedad inmobiliaria de forma vertiginosa en la época del boom inmobiliario 5455 .

Grafico 2.2. Participación de los segmentos más ricos en la riqueza total.

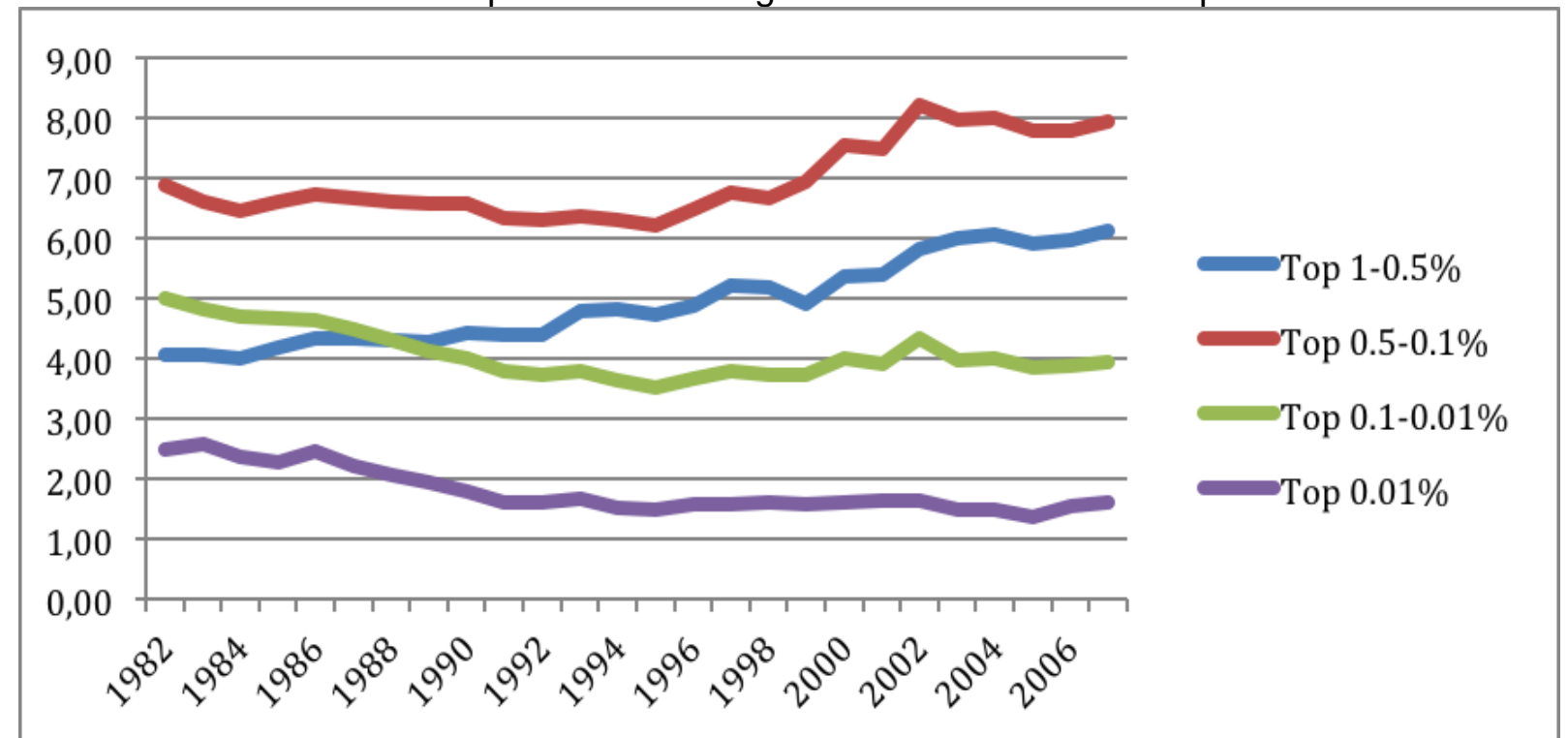

Fuente: Elaboración propia a partir de los datos de Income and Wealth Concentration in Spain from a Historical and Fiscal Perspective (tables and figures)

Como ya apunté anteriormente, la composición de la riqueza varía de forma sustancial conforme nos acercamos a los individuos más ricos (Tabla 2.1). Mientras que en el conjunto de la población el peso de los activos inmobiliarios es notablemente superior al resto de los componentes, conforme nos acercamos a los individuos con mayor riqueza este tipo de activos va perdiendo progresivamente importancia. De hecho, al analizar el 0,01 \% más rico, vemos como los activos

\footnotetext{
${ }^{53}$ Azpitarte, F. (2008)

54 Alvaredo, F. (2009) Pág. 1142

${ }^{55}$ Según los datos de la Sociedad de Tasación el incremento acumulado en términos reales del precio del $\mathrm{m}^{2}$ en España desde 1986 a 2007 fue del 260,43\%. Lo que supone un incremento medio anual ligeramente superior a 6 $\%$.
} 
Luque, Víctor. A propósito de Piketty: evolución de la desigualdad en España.

inmobiliarios dejan de ser el principal componente y su lugar lo ocupan las acciones bursátiles. Esta diferente composición nos ayuda a comprender la disminución de la participación de ese segmento en el total durante la época de incremento del precio de los activos inmobiliarios. Por el contrario, los incrementos en el valor de las acciones benefician notablemente más a los más acaudalados, haciendo que aumente -aún más- su participación en la riqueza total ${ }^{56}$.

Pese a que los datos analizados hasta 2007 puedan sugerir un enriquecimiento de las clases medias durante el período de mayor revalorización de los activos inmobiliarios, en la práctica el enriquecimiento no ha sido tal. Para aquellos que disponían de una única vivienda, la utilidad que les proporciona siguió siendo la misma pese a la revalorización. Al mismo tiempo, para aquellos individuos que no tuvieran vivienda en propiedad esta se convirtió en un bien casi inalcanzable. Con lo cual, aunque estadísticamente pueda parecer que se ha producido un estancamiento en la desigualdad de la riqueza, en la práctica supone un aumento de la desigualdad en las condiciones de vida.

En cualquier caso, la caída continuada del precio de los activos ${ }^{57}$ inmobiliarios desde 2007 habrá implicado un aumento de la participación de los segmentos más ricos de la población en la riqueza total, con la consiguiente caída en la participación de los segmentos más pobres. Y ello, porque el peso de los activos inmobiliarios en el conjunto de la riqueza es menor en los segmentos más ricos de la sociedad ${ }^{58}$. De hecho, la caída tan brusca que se ha producido de forma conjunta con que la inmensa mayoría de los préstamos hipotecarios contraídos no contemplaban la dación en pago, ha condenado a numerosas familias a perder no solo toda aquella riqueza que tenían, sino a quedarse con un saldo negativo debido a que el valor de la casa no cubría el valor de la deuda con el banco. Una medición del efecto que ha tenido la caída del precio de la vivienda en EEUU, tras la explosión de la burbuja inmobiliaria a partir de 2007, sobre los niveles de desigualdad de la riqueza la podemos encontrar en un reciente artículo de Atif Mian y Amir Sufi ${ }^{59}$. En él, se pone de manifiesto como la caída del precio de la vivienda ha ocasionado un aumento de la concentración de la riqueza en los segmentos más ricos de la población.

Durante el período considerado se han producido modificaciones sustanciales en los componentes de cada uno de los cuantiles poblacionales. Analizando la evolución de la composición durante el período en la riqueza del $1 \%$ más rico, observamos los siguientes elementos característicos: 1) Los activos inmobiliarios ganan participación durante el período en todos los segmentos poblacionales menos en el 0,01 \% más

\footnotetext{
${ }^{56}$ Alvaredo (2009) Pág. 1157.

${ }^{57}$ Según los datos de las Sociedad de Tasación desde diciembre de 2007 hasta diciembre de 2014 la caída en términos reales del precio medio del metro cuadrado de la vivienda libre en España ha sido del $-38,47 \%$. Una tasa de variación media anual del $-6,70 \%$.

${ }^{58}$ Azpitarte, F. (2008): "Households in the top decile present a much more diverse asset portfolio than that of other households. Indeed, other real estate, business equity, and stocks only have a significant weight in the portfolio of these households. For the middle class, by comparison, the main residence is by far the main asset (...)Vehicles, consumer durables, and collectibles are the main assets of the households in the bottom decile, as they account for almost 60 percent of the total assets of this group."

59 "Piketty and U.S. Wealth Inequality" disponible en http://houseofdebt.org/2014/05/24/piketty-and-u-s-wealthinequality.html. Para más información sobre este tema consultar el libro de estos mismos autores Mian, A., \& Sufi, A. (2014). House of Debt: How They (and You) Caused the Great Recession, and How We Can Prevent It from Happening Again. University of Chicago Press.
} 
Luque, Víctor. A propósito de Piketty: evolución de la desigualdad en España.

rico. 2) Se produce una caída generalizada del peso de los negocios en el total (caídas de alrededor del 80 \%). 3) El peso del efectivo, los depósitos y los bonos se reduce alrededor de un $50 \%$ para todos los segmentos analizados. 4) El componente que más participación gana son las acciones bursátiles.

Tabla 3.1. Composición de la riqueza de los segmentos de población más ricos:

Participación relativa en el total ${ }^{60}$.

\begin{tabular}{|c|c|c|c|c|c|c|c|}
\cline { 3 - 8 } \multicolumn{2}{c|}{} & $\begin{array}{c}\text { Activos } \\
\text { Inmobiliarios }\end{array}$ & Negocios & $\begin{array}{c}\text { Efectivo, depósitos y } \\
\text { bonos }\end{array}$ & Acciones & Otros & Deuda \\
\hline \multirow{2}{*}{ Top 1-0.5\% } & 1982 & 75,3 & 4,9 & 13,6 & 6,5 & 3,6 & $-3,9$ \\
\cline { 2 - 8 } & 2007 & 78,8 & 0,8 & 6,4 & 14,7 & 0,9 & $-1,5$ \\
\hline \multirow{2}{*}{ Top 0.5-0.1\% } & 1982 & 67,6 & 5,6 & 12,7 & 12,2 & 4,5 & $-2,6$ \\
\cline { 2 - 8 } & 2007 & 71,2 & 0,9 & 6,5 & 21,7 & 1,3 & $-1,7$ \\
\hline \multirow{2}{*}{ Top 0.1\%-0.01 \% } & 1982 & 55,8 & 5,2 & 11,3 & 24,7 & 5,6 & $-2,5$ \\
\cline { 2 - 9 } & 2007 & 58,0 & 0,8 & 6,9 & 34,3 & 2,0 & $-2,0$ \\
\hline \multirow{2}{*}{ Top 0.01\% } & 1982 & 36,8 & 2,9 & 10,7 & 46,2 & 5,6 & $-2,2$ \\
\cline { 2 - 8 } & 2007 & 27,4 & 0,5 & 5,8 & 65,9 & 3,6 & $-3,2$ \\
\hline
\end{tabular}

Fuente: Elaboración propia a partir de los datos de Income and Wealth Concentration in Spain from a Historical and Fiscal Perspective (tables and figures)

El estudio de la composición de la riqueza y de los ingresos por cuantiles nos permite prever a quién perjudica más (en proporción a su riqueza) una mayor presión fiscal sobre determinados activos y sobre determinadas rentas. Así, un análisis de determinadas propuestas de reforma tributaria o del actual modelo impositivo teniendo en cuenta las diferencias en las composiciones nos lleva a una configuración de las cargas tributarias mucho menos progresiva o incluso regresiva en los tramos más altos ${ }^{61}$.

\section{FUERZAS CONVERGENTES Y DIVERGENTES}

Como se comentó brevemente en la introducción, Piketty destaca varios elementos que actúan respecto a la distribución de los ingresos y la riqueza como fuerzas convergentes o divergentes. La principal fuerza convergente que señala es la de la difusión de los conocimientos. Como principales fuerzas divergentes destacan aquellas vinculadas al proceso de acumulación y de concentración de la riqueza en un mundo caracterizado por un bajo crecimiento y un elevado rendimiento del capital $(r>g)$ y por otro lado, al hecho de que las personas con una elevada remuneración pueden separarse del resto, rápidamente, por un amplio margen.

\footnotetext{
${ }^{60}$ Las "acciones" incluyen aquellas comercializadas en mercados abiertos como aquellas que lo hacen en mercados cerrados, en la categoría de "otros" están incluidos los seguros, anualidades y otros activos de menor importancia. Para más información vid. El apéndice de Alvaredo (2009).

${ }^{61}$ Vid. El Informe de la Comisión de Expertos para la Reforma del Sistema Tributario de 2014. En éste se incide, entre otras propuestas, en la conveniencia de: A) mantener el sistema dual de imposición, estableciendo para las rentas procedentes del ahorro un tipo único similar al mínimo de la tarifica progresiva existente para las rentas del trabajo. B) Establecer un tipo impositivo a los impuestos sobre sucesiones y donaciones reducido situado entre el $4-10 \%$. C) La supresión definitiva de los impuestos a los depósitos bancarios y D) el aumento de la tributación de inmuebles. Medidas todas que favorecen un menor gravamen sobre las rentas de capital que, como vimos, predominan en los ingresos de los que más ganan y sobre los activos típicos de los patrimonios más grandes.
} 
Luque, Víctor. A propósito de Piketty: evolución de la desigualdad en España.

Piketty expone, además, que estas fuerzas pueden producirse en un mundo en el que todas las inversiones adecuadas en competencias ya se hayan realizado, y en el que todas las condiciones de la eficacia de la economía de mercado -en el sentido de los economistas neoclásicos- aparentemente se hayan reunido.

En este apartado se analizará de forma breve la evolución que han seguido estos elementos o fuerzas, las previsiones de futuro, así como aquellos otros factores por los que se ven condicionados.

\section{DIFUSIÓN DE LOS CONOCIMIENTOS Y DE LA INVERSIÓN EN EDUCACIÓN, EN LA CAPACITACIÓN Y LA FORMACIÓN DE HABILIDADES}

Piketty describe este proceso como la principal fuerza de convergencia. Añade que puede verse favorecido por la apertura comercial, si bien consiste fundamentalmente en un proceso de difusión de los conocimientos y de compartir. Describe al saber como el bien público por excelencia y no como un mecanismo de mercado62.

Por otro lado, destaca que esta fuerza sólo es parcialmente natural y espontánea, puesto que está, en gran medida, condicionada o influida tanto por las instituciones que configuran el funcionamiento en el ámbito de la educación y de la investigación como por las políticas llevadas a cabo en éstas áreas ${ }^{63}$.

En la misma línea argumental se posicionan Martínez Celorrio, X. y Marín Saldo, A. (2012), cuando afirman que los factores institucionales son determinantes para hacer que funcione el ascensor social en economías globalizadas, turbulentas e inciertas. Las políticas redistributivas que disminuyen la desigualdad familiar de partida y las políticas educativas que amplían o restringen el acceso a las titulaciones más valiosas influyen en la eficacia de la educación como vehículo jerárquico de movilidad social.

Como vimos en el primer epígrafe, durante el período analizado no se ha producido una reducción en la desigualdad de los ingresos, sino que se ha dado una concentración en los segmentos más elevados de la distribución. En el mismo período, se ha producido un considerable incremento en la formación media de la población española ${ }^{64}$, por lo que, al menos aparentemente, el incremento educativo no ha sido capaz de compensar al resto de fuerzas de convergencia para que se haya producido una reducción en la desigualdad de los ingresos. Tampoco podemos achacar al cambio en la formación y habilidades medias el incremento de la desigualdad, puesto que de ser así, presumiblemente, esta concentración se habría dado en un grupo de población más amplio ${ }^{65}$. Los salarios de los puestos directivos $^{66}$ más elevados parecen ser más el resultado de pura suerte o de la

\footnotetext{
62 Piketty (2014) pág. 21

${ }^{63}$ Piketty (2014) pág. 22.

${ }^{64}$ Panorama de la educación. Indicadores de la OCDE 2013. Informe español. Instituto Nacional de Evaluación Educativa, Madrid, 2013.

${ }^{65}$ Syll, L. P. (2014) Pág. 37

${ }^{66}$ El estudio realizado por Fabbri, F y Marin, D. (2012) que analiza el notable aumento de los salarios de los directivos de las grandes empresas respecto al de los empleados en el período 1977-2009 en Alemania, llega a la conclusión de que los principales factores explicativos de este fenómeno son el poder de los directivos -en el sentido de que los ejecutivos buscan su propio interés y que son capaces de influir en el nivel de su
} 
Luque, Víctor. A propósito de Piketty: evolución de la desigualdad en España.

pertenencia al mismo club que aquellos que deciden sobre los salarios y los bonus que de lo que efectivamente aportan al proceso productivo ${ }^{67}$.

En este sentido, parece bastante evidente que el aumento retributivo de esa élite no puede explicarse sobre la base de un incremento diferencial de las productividades marginales 0 en los niveles educacionales de los diferentes segmentos poblacionales ${ }^{68}$. Conviene recordar que, pese a que la casuística a nivel internacional es diversa ${ }^{69}$, la diferencia salarial entre un alto directivo y un trabajador de nivel bajo ha pasado en los últimos 30 o 40 años de una ratio de 10-20 veces a otra de 100 o 200 veces.

Como anteriormente se menciona, durante las últimas décadas se ha producido un incremento en el nivel educativo. Sin embargo, tal y como pone de manifiesto el último Informe PISA publicado (2012), España aún se mantiene con unos niveles de rendimiento inferiores a la media de los países de la OCDE en todas las competencias analizadas (habilidad lectora, matemáticas y ciencias). Respecto al dominio del inglés, los españoles tampoco presentamos buenos resultados a nivel comparado: el último Eurobarómetro ${ }^{70}$ sitúa a España como el segundo país de Europa con menor nivel de inglés, solo por detrás de Hungría. Debemos tener en cuenta que, como pone de manifiesto el Informe Infoempleo Adecco 2013 ${ }^{71}$, el inglés es imprescindible tanto para acceder a un mayor número de puestos de trabajo como para las posibilidades de ascenso. Estas deficiencias o carencias educativas suponen un obstáculo para la igualdad de oportunidades educativas puesto que solo

\footnotetext{
compensación a expensas de los accionistas- y la competencia entre empresas a nivel nacional e internacional por los directivos. Sin embargo, rechazan la hipótesis de la eficiencia en el pago, es decir que el factor determinante del aumento salarial sea el incremento de su productividad marginal.

${ }^{67}$ Piketty (2014) Pág. 324: "Los datos que demuestran de la manera más convincente el fracaso de la "gobernanza de la empresa" y el hecho de que la fijación de las remuneraciones más altas tiene poco que ver con una lógica tradicional de productividad son los siguientes: cuando se reúnen bases de datos sobre empresas individuales (...) es muy difícil explicar las variaciones observadas de las remuneraciones de los directivos en función de los resultados de las empresas consideradas. Más precisamente, se puede desglosar cierto número de indicadores de rendimiento - el crecimiento de las ventas de la empresa, el nivel de sus beneficios, etc. — en las variaciones provocadas por causas externas a la empresa (...)y el resto de las variaciones. Sólo las variaciones del segundo tipo pueden verse potencialmente afectadas — por lo menos en parte- por los directivos de la empresa. Si las remuneraciones siguieran la lógica de la productividad marginal, podríamos esperar que no variaran - o poco - en función del primer componente, y sólo —o sobre todo- en función del segundo. Ahora bien, el hecho es que se observa exactamente lo contrario".

${ }^{68}$ En 1960, el salario medio, después de impuestos, de un director ejecutivo de una de las mayores empresas de Estado Unidos era 12 veces mayor que el salario medio de un trabajador fabril. En 1974, el salario más las bonificaciones que recibía un director general habían aumentado hasta equivaler a 35 veces el salario de un trabajador medio de su compañía. En 1980, un director general medio ganaba ya 42 veces más dinero que un trabajador manual medio, y esta cifra se dobló diez años más tarde al llegar a 84 veces su salario. Y entonces, entorno a 1980, se produce una hiperaceleración de la desigualdad. A mediados de la década de los años 90 , según el Business Week, la diferencia era ya de 135 veces; en 1999 ya había alcanzado el umbral de las 400 veces y en el año 2000 subió hasta 531. Vid. En Estudios citados en "Explorations in Social Inequality" < http://www.trinity.edu/mkearl/strat.html> [acceso: marzo 2015].

${ }^{69}$ Según un reciente artículo de The Economist que utiliza datos de la OCDE y Eurostat, España en 2012 se situaba como el cuarto país europeo con mayores diferencias salariales entre trabajadores y directivos, solo por detrás de Rumanía, Ucrania y Rusia. http://www.economist.com/blogs/graphicdetail/2013/06/daily-chart$6 ? \mathrm{fsrc}=\mathrm{scn} / \mathrm{fb} / \mathrm{wl} / \mathrm{dc} /$ weekswages

${ }^{70}$ Special Eurobarometer 386 EUROPEANS AND THEIR LANGUAGES REPORT (2012) Disponible en: http://ec.europa.eu/public_opinion/archives/ebs/ebs_386_en.pdf, última vez consultado 1 Junio 2015.

${ }^{71}$ Disponible en http://blog.infoempleo.com/wp-

content/uploads/2014/07/Informe_Infoempleo_Adecco_2013.pdf, consultado el 1 de Junio de 2015.
}

Papeles de Europa

Vol. 28, Núm. 1 (2015): 86-115

http://dx.doi.org/10.5209/rev_PADE.2015.v28.n1.50182 
Luque, Víctor. A propósito de Piketty: evolución de la desigualdad en España.

podrán suplirlas mediante el envío de los hijos a estudiar inglés en el extranjero o en academias privadas aquellas familias con un nivel de ingresos o de riqueza elevado.

A pesar de lo expuesto, es interesante preguntarnos cómo ha influido el aumento de la formación en la movilidad social. Un reciente estudio sobre la evolución del grado de movilidad y la reproducción social que se da en España como consecuencia de la educación ${ }^{72}$, señala varios aspectos interesantes sobre esta cuestión:

- Comparando la población nacida en 1950-59 con la nacida entre 1970-79, se observa una mejora en la evolución de las oportunidades relativas de obtención de niveles de educación terciaria para aquellos individuos cuyos progenitores carecían de este grado educativo ${ }^{73}$. Aún así, las posibilidades de alcanzar este grado educativo pueden variar más del doble, en función del nivel educativo de los progenitores. El valor de este ratio ${ }^{74}(2,3)$ está en la media de la UE-25, mientras que países como Polonia o Italia presentan valores mucho más elevados (7,5 y 6,3 respectivamente) y otros como Finlandia y Gran Bretaña, valores inferiores (1,5 y 1,8 respectivamente).

- Destaca la escasa movilidad que caracteriza a las clases bajas y a la del extremo superior en comparación con la clase media. Para los cohortes de edad estudiados, las clases superiores muestran un cierre social de alrededor del $60 \%$, las clases bajas cercano al $55 \%$, mientras que los orígenes de clases medias han mantenido constante una herencia o inmovilidad próxima al 15\%.

- La influencia del origen social sobre los destinos de clase de los hijos sólo afecta a los bajos niveles educativos, evidenciando el círculo cerrado de reproducción y herencia social por abajo. Sin embargo, el origen social no afecta al enclasamiento de los hijos con niveles secundarios y universitarios.

Estos datos corroboran el papel determinante de la educación como uno de los principales factores de estratificación, movilidad y enclasamiento. Este mismo estudio alerta del peligro de inversión de la tendencia al aumento de la movilidad como consecuencia del cambio de modelo al que se está tendiendo y de los recortes que está sufriendo la educación pública. Las últimas reformas educativas avanzan a un modelo en el que el Estado garantiza una formación básica, cada vez con menor peso, mientras que se prima los másteres y postgrados a la hora de la inserción laboral en los puestos más altos. Por tanto, se crea una reducción en las posibilidades de ascenso social, en la medida que el precio de estos títulos es considerablemente superior al de las antiguas licenciaturas 0 al de los actuales grados $^{75}$.

\footnotetext{
${ }^{72}$ Martínez Celorrio (2013).

${ }^{73}$ En España hemos pasado de un 16\% de hijos con padres de educación básica que logra titulaciones superiores y terciarias en los nacidos entre 1950-59 a un 33\% entre los nacidos entre 1970-79 con los mismos antecedentes familiares. Mientras que hemos pasado de un $69 \%$ a un $73 \%$ de herencia de la educación superior o terciaria entre padres e hijos en estos últimos 20 años.

${ }^{74}$ Probabilidad de logro de niveles de educación terciaria de los hijos según el nivel educativo superior/básico del padre.

${ }^{75}$ Los datos de la estadística Universitaria del Ministerio de Educación, Cultura y Deporte dejan patente cómo la pérdida de matriculados en las Universidades se produce en el mismo lustro en el que los precios por crédito suben, de media, un $45 \%$.

http://www.elconfidencial.com/espana/2014-07-31/estudiar-cuesta-mas-el-precio-de-los-creditos-universitariossube-un-45-desde-2009_170428/
} 
Luque, Víctor. A propósito de Piketty: evolución de la desigualdad en España.

Además, en los últimos años se ha producido un aumento significativo de los precios de las primeras matrículas y aún mayor en el de las siguientes. Este incremento no se ha dado de forma homogénea en el conjunto de regiones en España, sino que existen claras diferencias, habiendo aumentado considerablemente la distancia entre las comunidades donde las matrículas son más baratas de aquellas en las que el coste es mayor ${ }^{76}$. Unas diferencias que, como reconocen los informes elaborados por la Conferencia de Rectores de las Universidades Españolas sobre el estado de la educación, no parecen responder a retornos diferenciados en términos de cantidad o de calidad de los servicios universitarios prestados, sino a exclusivos criterios de territorialidad y a las distintas políticas aplicadas en las comunidades autónomas y de los que derivan indudables elementos de segmentación e inequidad en el seno del sistema universitario español. Los efectos de esta subida de los precios públicos se ven agravados por su coexistencia con una tendencia a la reducción de las ayudas al estudio y de los sujetos susceptibles de recibirlas de forma paralela con un aumento de los demandantes, consecuencia del deterioro de la situación económica de las familias ${ }^{77}$.

Por otra parte, debemos ser conscientes que no es suficiente con el establecimiento de un sistema universal de educación básica. Esta cuestión no se puede asimilar a un acceso equitativo, puesto que la clase social de origen condiciona de manera desigual el aprovechamiento de los recursos formativos públicos, el acceso a los recursos formativos privados y la propia capacidad del entorno familiar -sobre todo de los padres- para acompañar y apoyar el proceso educativo de los alumnos. En definitiva, el fracaso y el abandono escolar no afectan por igual a las diferentes clases sociales ${ }^{78}$.

Los factores institucionales son determinantes para hacer que funcione el ascensor social en economías globalizadas, turbulentas e inciertas. Las políticas redistributivas que disminuyen la desigualdad familiar de partida y las políticas educativas que amplían o restringen el acceso a las titulaciones más valiosas influyen en la eficacia de la educación como vehículo jerárquico de movilidad social $^{79}$.

\section{EL CRECIMIENTO DEMOGRÁFICO}

El diferente ritmo de crecimiento demográfico tiene implicaciones importantes en la evolución de la concentración de la riqueza personal. Permaneciendo el resto de cosas constantes, un fuerte crecimiento demográfico tiene un efecto igualador, puesto que reduce la importancia de la herencia: "cada generación debe en cierto sentido construirse a si misma" 80 .

Los elementos determinantes del crecimiento demográfico son la esperanza de vida, la tasa de natalidad y los flujos migratorios. A su vez, la evolución de estas variables

\footnotetext{
${ }^{76} \mathrm{Si}$ se compara el curso académico 2012/13 con el curso 2009/10 en el caso de las primeras matrículas se observa que la diferencia entre la de más bajo coste, Andalucía, y la de más alto, Aragón, era entonces de 10,82 euros y ha pasado ahora a una diferencia de 26,04 euros entre Andalucía y Cataluña

${ }^{77}$ Michavila, F. (2013) Pág. 203.

${ }^{78}$ Martínez-Celorrio, X., \& Marín Saldo, A. (2012) Pág. 120.

${ }^{79}$ Ibíd. Pág. 121.

${ }^{80}$ Piketty (2013) Pág. 83.
} 
depende de numerosos factores. La esperanza de vida está condicionada -entre otros- por circunstancias sanitarias, factores ambientales, hábitos de vida e ingresos medios. La tasa de natalidad se ve influida por factores culturales, económicos, psicológicos y personales, los cuales dependen, al mismo tiempo del grado de conciliación de la vida familiar y laboral permitido por el marco institucional. Por último, los flujos migratorios se ven influidos por factores de naturaleza geográfica, política, económica y cultural.

Gráfico 3.1: Pirámide de población española: años 2013 y 2023

Pirámide de población. Años 2013 y 2023

España

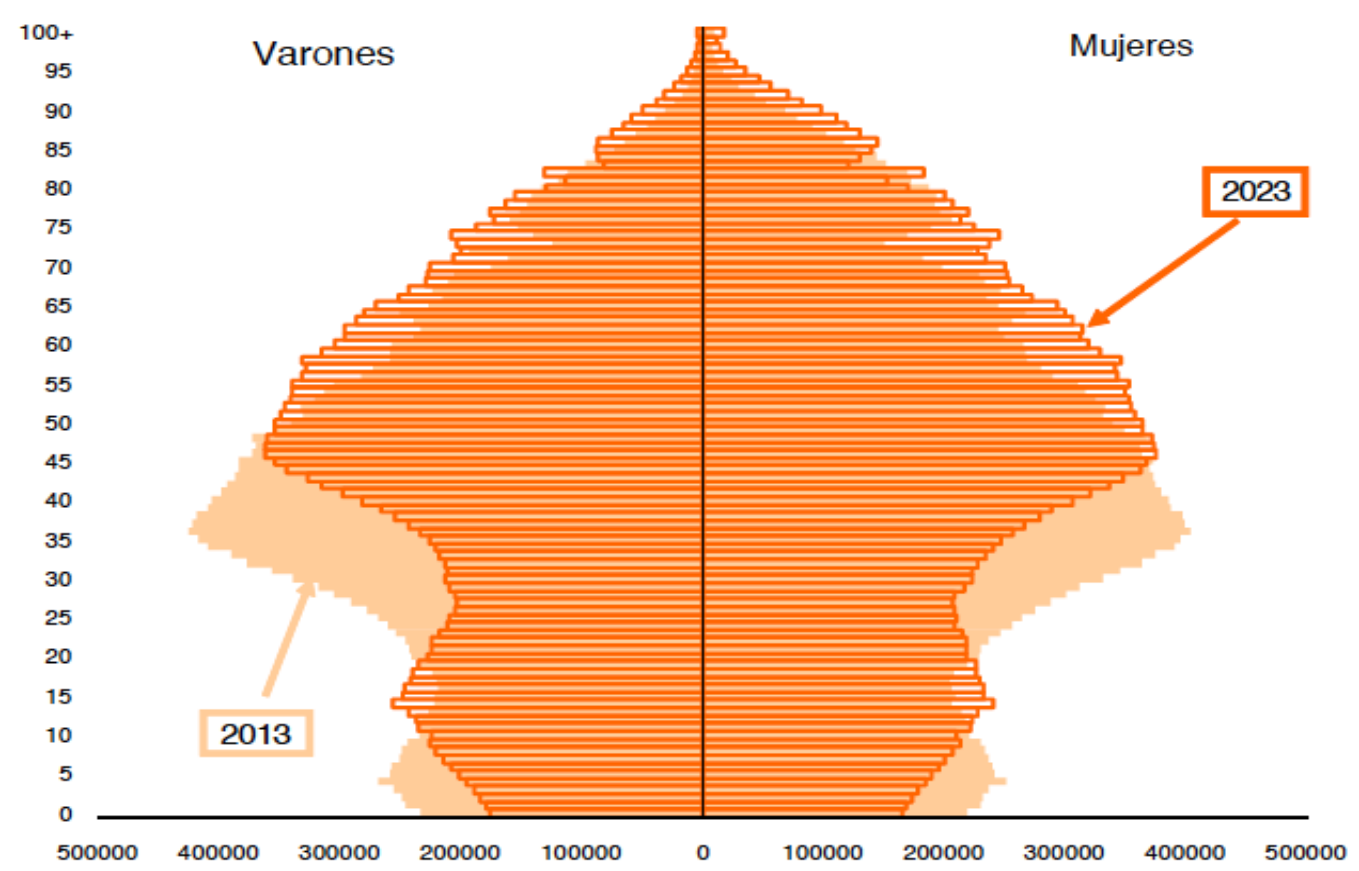

Fuente: Gráfico extraído del INE: “Proyección de la población de España a Corto Plazo 2013-2023"

Sobre esta cuestión se debe advertir que, cuando Piketty alude al crecimiento demográfico, no se refiere al crecimiento a través de los flujos migratorios. Este aspecto lo trata brevemente casi al final del libro, señalando que dejar que el trabajo se desplace hacia lugares con salarios más altos es otra forma de redistribuir y de regular la desigualdad mundial del capital. Sin embargo, también se refiere a las limitaciones que presenta esta variable del crecimiento, que, según él, solo explica una parte del problema de la desigualdad. Según el citado autor, "Una vez que la producción y los ingresos promedio de los países se igualan por la inmigración y, sobre todo, porque la productividad de los países pobres se acerca a la de los ricos, siguen los problemas planteados por las desigualdades y, en particular, por la dinámica de concentración de los patrimonios en el ámbito mundial. La redistribución por inmigración no hace más que posponer un poco más el problema, pero no exime de instituir las regulaciones necesarias -estado social, impuesto progresivo sobre el ingreso e impuesto progresivo sobre el capital-"81.

\footnotetext{
${ }^{81}$ Piketty (2014) Pág. 530.
} 
Luque, Víctor. A propósito de Piketty: evolución de la desigualdad en España.

Según lo anteriormente expuesto, se puede concluir que los impactos que presentan sobre la desigualdad, tanto los flujos migratorios como el aumento de la tasa de natalidad serán diferentes. Solo unas elevadas tasas de natalidad harán que se reparta -aunque sea entre los propios hijos- la riqueza intergeneracional. El crecimiento demográfico explicado principalmente por flujos migratorios positivos y combinado con unos bajos índices de natalidad no hace que este instrumento entre en funcionamiento, sino que, por el contrario, podría conducir a mayores niveles de desigualdad tanto en la riqueza como en los ingresos, a menos que existan unas instituciones adecuadas -Estado social, normativa laboral, etc.- que compensen la presión a la reducción salarial que puede provocar esta cuestión. En definitiva, la riqueza pasará de una generación de nacionales a otra de forma muy concentrada, debido a las bajas tasas de natalidad y a la propia dinámica de concentración del capital. Consecuentemente, la distancia entre este grupo privilegiado y la mayoría de nacionales, que apenas tienen participación en la riqueza irá en progresivo aumento, distancia que podría ser mayor incluso en el caso de los inmigrantes. Por la parte de los ingresos, el incremento de la oferta de trabajo -provocado por la llegada de inmigrantes en edad laboral y con baja formación- podría ocasionar una presión a la baja sobre los salarios de los trabajadores lo cual, presumiblemente, potenciaría la tendencia al aumento de la desigualdad en los ingresos.

Otro aspecto que sería interesante tener en cuenta es el hecho de que la tasa de natalidad no tiene por qué ser homogénea a medida que nos desplazamos por los diferentes segmentos de población con mayores o menores niveles de riqueza. Si dicha tasa de natalidad es superior en los segmentos poblacionales con niveles de riqueza más bajos respecto a aquellos más ricos, las desigualdades se amplificarán con el paso de las generaciones. Piénsese que en esta hipótesis, los pequeños patrimonios se deberán repartir entre más sujetos por termino medio, mientras que los patrimonios superiores se distribuirán entre menos sujetos. De darse el caso contrario -tasa media más baja en los segmentos más desfavorecidos y más alta en los segmentos más ricos- el efecto sería lógicamente diferente, al favorecer un reparto de la riqueza más equitativo. En cualquier caso, dadas las limitaciones de este estudio no podemos profundizar más en esta cuestión.

El Instituto Nacional de Estadística (en adelante INE) prevé, en lo que a proyección de la población de España se refiere, una reducción del número de nacimientos en los próximos años ${ }^{82}$. Ello, pese a contemplar un aumento del número medio de hijos por mujer, que se estima en 1,41 en 2022, frente al 1,34 actual. Según los datos del citado organismo, la esperanza de vida al nacimiento alcanzará 87 años las mujeres y 81,8 años en los varones. Del mismo modo, se indica que el número de defunciones se incrementará como consecuencia del envejecimiento poblacional. A este respecto, se advierte que la conjunción descenso en la natalidad y envejecimiento poblacional provocará que, en 2017, haya más defunciones que nacimientos, alcanzando un saldo vegetativo negativo. En lo que se refiere a los flujos migratorios, se contempla una consolidación de los saldos negativos que se vienen produciendo desde 2010, al menos hasta 2022 (último año de proyecciones).

\footnotetext{
${ }^{82}$ Nota de prensa del INE de 22 de noviembre de 2013. "Proyección de la población de España a Corto Plazo 2013-2023”.
} 
Luque, Víctor. A propósito de Piketty: evolución de la desigualdad en España.

De cumplirse estas estimaciones, podría decirse que la evolución demográfica de España se configurará como una fuerza divergente, reforzando la tendencia al aumento de la desigualdad.

\section{EL CRECIMIENTO ECONÓMICO}

Los efectos de un rápido crecimiento económico se asemejan a los de un fuerte crecimiento demográfico, puesto que reduciría la importancia del capital acumulado por anteriores generaciones. Además, con bajas tasas de crecimiento es bastante posible que $r>g$.

Piketty identifica, además, otro mecanismo que puede contribuir a la reducción de la desigualdad: un crecimiento constante implica la continua creación de nuevas funciones y habilidades por cada generación lo que favorece la movilidad social ${ }^{83}$. Este incremento de la movilidad social no conlleva por sí solo, necesariamente, una reducción de la desigualdad de ingresos, pero teóricamente limita la reproducción y amplificación de las desigualdades de riqueza y por tanto, en el largo plazo, también limita la desigualdad de ingresos. Al menos de aquellos derivados del capital.

Para una mejor comprensión del proceso de crecimiento económico, Piketty lo desagrega en crecimiento de la población y crecimiento de la producción per cápita, de modo que podamos distinguir un componente puramente demográfico y otro específicamente económico. Este último es el que permite la mejora de las condiciones de vida. Así, el crecimiento de la producción vendría explicado por la siguiente fórmula:

$$
g=p+(g / p)
$$

Donde $g$ representa la tasa de crecimiento (es decir, el incremento anual del ingreso y la producción) y $p$ simboliza la tasa de variación anual de la población.

A lo largo del período 1980-2014, el PIB de la economía española ha crecido a un ritmo medio anual del $2,26 \%$, el cual ha venido explicado por un crecimiento medio anual de la población del 0,59 \% y un aumento del PIB per cápita del 1,36 \% medio anual. Ahora bien, el crecimiento de ambos componentes no ha sido constante a lo largo del período, como se observa en el gráfico 3.2. El crecimiento de la población ha experimentado tasas positivas durante todo el período, hasta llegar a 2013, año en el que se inicia una caída en el número de personas que residen en España, la cual se espera que continúe en los próximos años. La etapa de mayor crecimiento de la población se dio entre el año 2002 y 2008 (1,9\% de incremento medio anual), debido principalmente a los flujos migratorios positivos, consecuencia de la época de expansión económica. Las fluctuaciones en el PIB per cápita han sido más acusadas, presentando variaciones negativas en los años 1981, 1993 y durante el período 2009-2013, con la excepción del 2010, en el cual prácticamente se mantuvo el nivel de producción del año anterior.

\footnotetext{
${ }^{83}$ Piketty (2014) Pág. 85.
} 
Luque, Víctor. A propósito de Piketty: evolución de la desigualdad en España.

Grafico 3.2 Variación del PIB de la economía española: Descomposición en variación de la población y PIB per cápita. Datos en términos porcentuales.

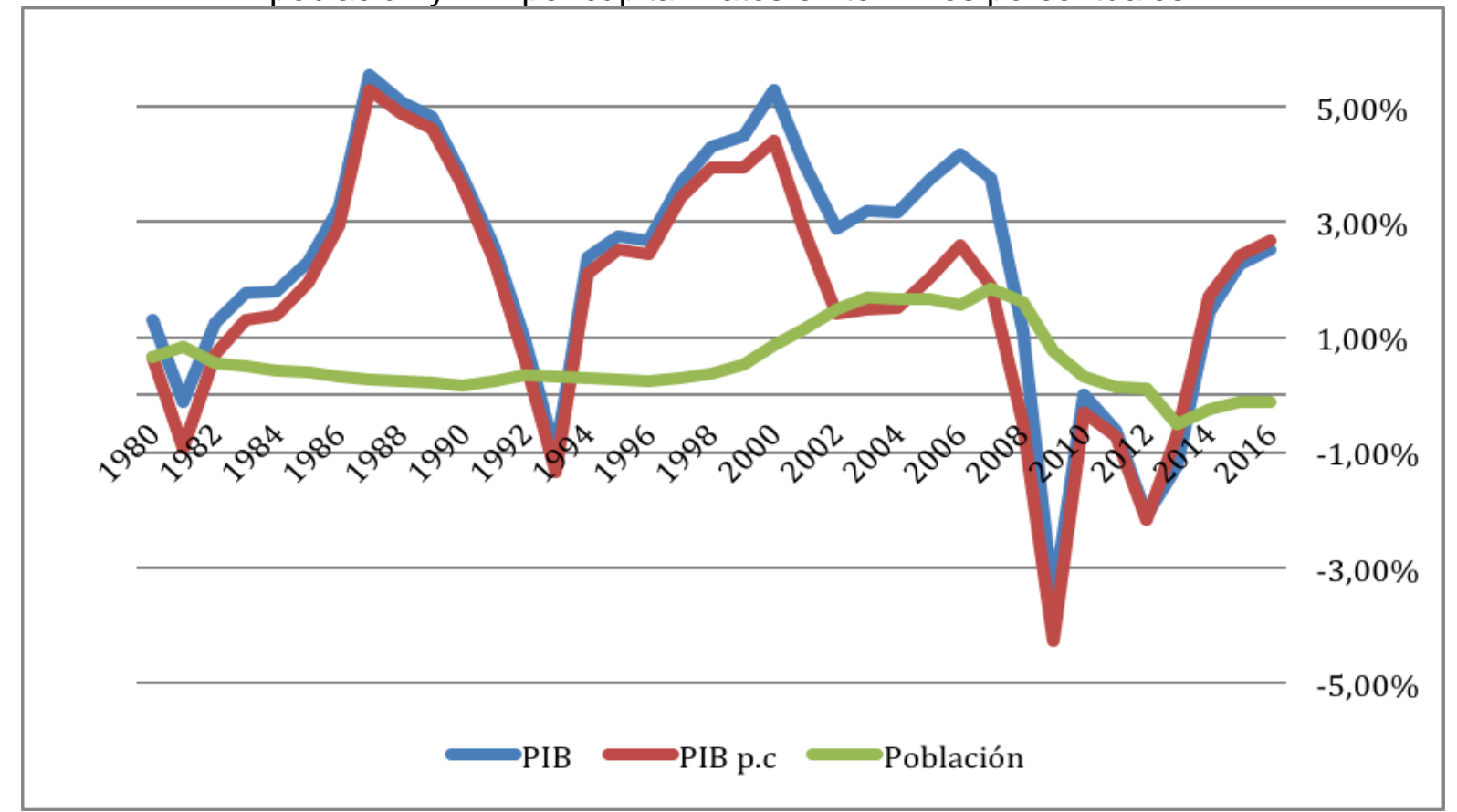

Fuente: Elaboración propia a partir de AMECO data base. Los datos de 2015 y 2016 son las previsiones que maneja AMECO.

Puesto que el componente demográfico ha sido abordado en el anterior epígrafe, a continuación nos centraremos en la evolución del PIB per cápita. Para un mejor entendimiento del comportamiento de éste, vamos a desagregarlo, a su vez, en el crecimiento de la productividad por trabajador y en el del número de trabajadores, en relación con la población:

$$
\mathrm{g} / \mathrm{p}=\mathrm{g} / \mathrm{l}+\mathrm{l} / \mathrm{p}
$$

Dónde hemos agregado a la fórmula anterior (1) "l" , que representa la tasa de variación anual del número de trabajadores.

El notable crecimiento del PIB per cápita que experimentó la economía española desde la década de los 80 -con la única excepción del año 1993 con tasas de variación negativa- se trunca bruscamente con la fuerte crisis que se inicia en 2008 , en la cual el PIB per cápita sufrió un retroceso acumulado (hasta 2013) de casi el 8 \%. En el año 2014 se produce un incremento, situándose no obstante en valores absolutos considerablemente inferiores a los del comienzo de la recesión. El crecimiento del PIB per cápita que se ha dado a lo largo del período analizado $(1,65$ $\%$ medio anual) viene explicado por un crecimiento medio anual de la productividad del $1,36 \%$ y un crecimiento medio del ratio trabajadores/población del 0,29 \%. Sin embargo, en las épocas de mayor crecimiento del PIB per cápita, este viene explicado principalmente por un aumento del ratio L/población, mientras que en las épocas de caída en el PIB per cápita, las ganancias de productividad habrían compensado (aunque solo parcialmente) la caída del ratio. Según lo expuesto, los aumentos de productividad más altos se habrían producido en los momentos de descenso del porcentaje de población trabajadora, efecto de la caída de $L$ en mayor cuantía que la del PIB. 
Gráfico 3.3 Evolución de la productividad y del ratio trabajadores ${ }^{84} /$ población

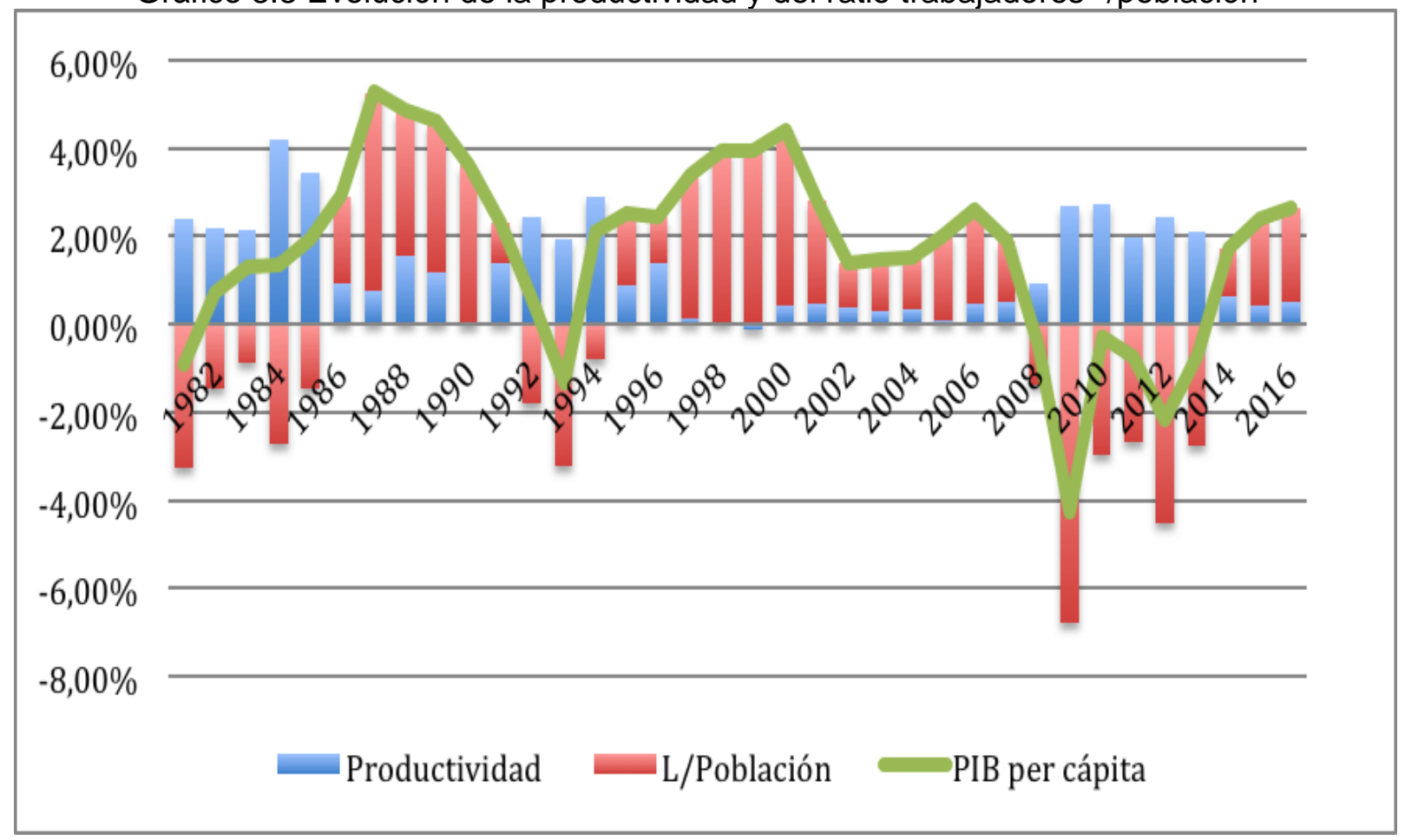

Fuente: Elaboración propia a partir de los datos de AMECO

Ahora bien, mientras que la aportación de la productividad ha sido positiva (o como mínimo nula) en todos los años que estamos estudiando, la ratio L/Población ha fluctuado de forma mucho más brusca, con períodos de fuerte creación de empleo y otros de elevada destrucción. Si desde 1995 a 2007 se crearon más de 6,5 millones de puestos de trabajo en la economía española, desde 2008 hasta 2014 se han destruido cerca de 3,5 millones.

Las previsiones del FMI sobre el crecimiento de la economía española en los próximos años son relativamente positivas ${ }^{85}$. Sin embargo, esta evolución viene parcialmente explicada por el efecto rebote de tantos años de sucesivas caídas. Además, las perspectivas de crecimiento internacionales y en particular de la Unión Europea no son excesivamente halagüeñas ${ }^{86}$, por lo que previsiblemente el crecimiento a largo plazo será menor que el incremento medio experimentado en las últimas décadas. Así el efecto atenuante sobre la desigualdad que presumiblemente ha tenido el crecimiento de la economía española durante las últimas décadas será inferior en el futuro.

\footnotetext{
${ }^{84}$ Durante todo el apartado nos referimos a empleo a tiempo completo equivalente. Es decir, la existencia de dos puestos de trabajo de 4 horas diarias sería contabilizada de forma equivalente a la de uno que fuera de 8 horas al día.

${ }^{85}$ Variación del PIB en 2015 de 1,8 \% y de 1,7 \% en 2016. Vid. FMI: Perspectivas de la economía mundial Contracorriente 20 Enero 2015.

${ }^{86}$ El último informe del FMI sobre las previsiones de crecimiento realiza previsiones a la baja para las economías de China, Rusia y los países exportadores de materias primas. Respecto al impacto de la caída del precio del petróleo sobre la economía mundial señala que: "La caída de los precios del petróleo — producida en gran medida por el aumento de la oferta - estimulará el crecimiento mundial. Pero, según las proyecciones, ese estímulo se verá ampliamente superado por factores negativos; uno de ellos será la debilidad de la inversión a medida que muchas economías avanzadas y de mercados emergentes continúen adaptándose a un crecimiento a mediano plazo que ofrece expectativas menos alentadoras". Vid Ídem.
} 
Luque, Víctor. A propósito de Piketty: evolución de la desigualdad en España.

A todo lo expuesto habría que añadir que, en nuestra opinión -Piketty no se centra sobre este aspecto-, el modelo de crecimiento que ha emprendido España como salida a la crisis coadyuva en menor medida -en comparación con otro modelo que permitiese experimentar tasas de crecimiento similares- a la reducción de la desigualdad. Tratar de salir de la crisis siendo más competitivos en costes, por un lado disminuye directamente los salarios -que como vimos suponen el principal componente de los ingresos de los deciles más bajos, en comparación con los de mayores ingresos- y por otro, no favorece precisamente la creación de nuevas funciones, la innovación en productos o la especialización en ramas productivas de mayor valor añadido e intensidad tecnológica.

Por último, debemos recordar que el crecimiento del PIB per cápita en el largo plazo, como hemos visto anteriormente, viene explicado principalmente por el incremento de la productividad. Un modelo de crecimiento con una reducción del gasto en I+D+i es probable que limite el aumento potencial de la productividad, dado que los sectores con mayor contenido tecnológico son más proclives a experimentar tasas superiores de incremento de la productividad ${ }^{87}$.

\section{CONCLUSIONES}

Durante el período 1981-2012, se ha producido un aumento de la concentración de los ingresos en los segmentos más ricos de la población española (Top 1\%). Consecuentemente, el resto de percentiles, incluso aquellos situados entre el percentil 10 y el $1 \%$, ha visto reducida su participación en los ingresos. El incremento más significativo lo encontramos en el 0,01 \% que más ingresa de la población, el cual multiplicó por más de cuatro veces su peso en el ingreso nacional entre los años 1981 y 2006.

En cuanto a la composición media de los ingresos durante el citado período, hemos observado que, a medida que nos concentramos en los segmentos que más ingresan, el porcentaje de rentas procedentes del trabajo va disminuyendo y su lugar lo ocupan las que proceden del capital.

Respecto a la evolución de la riqueza en España durante el período 1981-2007, se observa un incremento sustancial de la misma (de casi el $5 \%$ medio anual). La categoría que más aumentó, en términos absolutos, fue la de los activos inmobiliarios, mientras que, en términos relativos, se incrementó más el valor de las acciones empresariales. A lo largo de la distribución de la población por riqueza, observamos que se produce una notable modificación en la composición típica de los patrimonios: mientras que, en el conjunto de la población, el peso de los activos inmobiliarios es superior al resto de componentes, conforme nos acercamos a los individuos con más riqueza, este tipo de activos va perdiendo importancia y su lugar lo ocupan las acciones bursátiles.

\footnotetext{
${ }^{87}$ Como ejemplo se puede destacar el crecimiento que experimentó Alemania durante el período 1995-2007. En este período aumentó el peso de las manufacturas con alto contenido tecnológico en la economía y además fueron las que experimentaron mayores tasas de crecimiento tanto de la productividad como de los salarios de los trabajadores. Vid. Luque, V. (2014)
} 
Luque, Víctor. A propósito de Piketty: evolución de la desigualdad en España.

Esta diferente composición ha provocado que, en el período analizado, si bien sí se ha producido un aumento de la participación del primer decil en su conjunto (pasando del 10,95 \% al 14,07\%), el $1 \%$ con mayor riqueza ha visto reducido su peso. Sin embargo, dada la evolución de los precios de los diferentes activos que se ha dado desde entonces, presumimos que la concentración de la riqueza se habrá acentuado, tanto en el primer percentil, como en el primer decil.

El estudio de las principales fuerzas convergentes y divergentes (difusión de los conocimientos y de inversión en educación, evolución demográfica y ritmo de crecimiento económico) no es especialmente alentador. Si bien han tenido un desempeño positivo en las décadas estudiadas, éste no ha sido suficiente para compensar la tendencia al incremento de la desigualdad. Ello nos hace suponer que la evolución prevista para los próximos años creará aún más presiones a favor del aumento en la concentración, tanto de ingresos, como de riqueza.

Según lo expuesto, puede parecer que, a veces, se cae en un cierto determinismo sobre el crecimiento de la desigualdad. Sobre este aspecto, Piketty plantea que, en el marco institucional neoliberal, de no darse fuertes shocks externos y siendo previsible que $r>g$, se producirá un incremento de la desigualdad. Todo ello, en el ámbito de un tipo de regulación que protege preferentemente los derechos de propiedad y de sucesión, a la vez que facilita la concentración de capitales, con ordenamientos tributarios regresivos cuando nos acercamos a los tramos más altos, así como con un funcionamiento de los bancos centrales más centrados más en el control de los precios que en el crecimiento equilibrado y en la corrección de las desigualdades. Sin embargo, como se mencionó en la introducción, a lo largo del libro y en trabajos posteriores, Piketty matiza la importancia de $r>g$, señalando que:

El resultado de esta historia dependerá de las representaciones que se hacen las diferentes sociedades de las desigualdades, y de las políticas e instituciones que se atribuyen para modelarlas y transformarlas, en uno u otro sentido ${ }^{88}$.

Llegados a este punto, debería de plantearse la imperiosa necesidad de corregir el actual marco institucional en aras de la consecución de unos mayores niveles de igualdad. Ello implicaría, entre otras medidas: a) el establecimiento de un marco tributario supranacional progresivo, y b) el impulso de un sistema educativo universal dirigido a promover, tanto un desarrollo productivo basado en el conocimiento y en la generación de bienes y servicios de mayor valor añadido, como un desarrollo personal, en igualdad de oportunidades al conjunto de la población. Además, ante los niveles de desigualdad y pobreza existentes, debería plantearse la implantación de mecanismos de urgencia, a corto plazo, que palíen la situación en la que vive un elevado porcentaje de nuestra población.

Por ultimo, considero necesario aclarar el alcance limitado de trabajo. Es un sencillo ejercicio de acercamiento a temas fundamentales sobre la economía española del cual surge una amplia "agenda de trabajo" para el futuro. Se necesita:

- Profundizar el análisis con datos con un mayor grado de desagregación territorial.

- Tratar de ampliar el período de estudio mediante la utilización de datos fiscales para la estimación de los indicadores de desigualdad.

88 Piketty (2014) Pág. 36

Papeles de Europa

Vol. 28, Núm. 1 (2015): 86-115

http://dx.doi.org/10.5209/rev_PADE.2015.v28.n1.50182 
Luque, Víctor. A propósito de Piketty: evolución de la desigualdad en España.

- Relacionar la distribución con la evolución de los diferentes componentes de la demanda agregada.

- Análisis más detallado de los efectos de la financiarización sobre la distribución.

- Relacionar el tema de la distribución con la balanza comercial, la competitividad exterior y la inserción exterior.

- Analizar el crecimiento y su reparto entre los diferentes cuantiles de renta y de riqueza en diferentes subperíodos relacionándolo con el contexto internacional y los cambios institucionales.

- Respecto a factores como la productividad, tratar de observarla desagregada por ramas productivas.

- Analizar de forma desagregada la distribución de los diferentes tipos de ingresos y de riqueza, de manera que permita una mejor identificación de los grupos poblacionales a los que afecta con mayor o menor intensidad las diferentes reformas tributarias.

- Incluir la evolución de la inflación como factor condicionante de la evolución de los distintos tipos de desigualdades. 
Luque, Víctor. A propósito de Piketty: evolución de la desigualdad en España.

\section{BIBLIOGRAFÍA}

Alvaredo, F., y Saez, E. (2009): "Income and wealth concentration in Spain from a historical and fiscal perspective", Journal of the European Economic Association, 7(5), 1140-1167.

Alvaredo, F., Atkinson, A. B., Piketty, T., \& Saez, E. (2013): "The top 1 percent in international and historical perspective", en National Bureau of Economic Research, 3-20.

Azpitarte, F. (2008): "The household wealth distribution in Spain: The role of housing and financial wealth", en XV Encuentro de Economía Pública: políticas públicas y migración, 38-53.

Bartels, L. M. (2009): "Unequal democracy: The political economy of the new gilded age", Princeton University Press, Princeton.

Bauman, Z. (2014): “¿La riqueza de unos pocos nos beneficia a todos?”, Paidós, Barcelona.

Bourguignon, F. (2013): "La mondialisation de l'inégalité”, Seuil, París.

Comisión de Expertos Para la Reforma del Sistema Tributario Español, "Informe 2014".

Eurobarómetro (2012): "Especial Eurobarometer 386: Europeans and their languages report", Junio 2012. Disponible en: http://ec.europa.eu/public_opinion/archives/ebs/ebs_386_en.pdf, última vez consultado 1 Junio 2015.

Fabbri, F., y Marin, D. (2012): "What Explains the Rise in CEO Pay in Germany? A Panel Data Analysis for 1977-2009" CEPR Discussion Paper No. DP8879.

Gilens, M. (2012): "Affluence and influence: Economic inequality and political power in America", Princeton University Press, Princeton.

Jayati Ghosh (2014): "Piketty and the resurgence of patrimonial capitalism". realworld economics review, issue no. 69, 7 Oct 2014,138-144.

Infoempleo, Adecco (2013): "Informe Infoempleo Adecco: Oferta y Demanda de Empleo en España", Madrid, 2013. Disponible en: http://blog.infoempleo.com/wp-

content/uploads/2014/07/Informe_Infoempleo_Adecco_2013.pdf, última vez consultado 1 Junio 2015.

Kuznets, S. (1955): "Economic Growth and Income Inequality", The American Economic Review, American Economic Association, vol. 45, núm. 1, marzo de $1955,1-28$.

Lucas, R. E. (2002): "The industrial revolution: Past and future", Lectures on economic growth, 109-188.

Luque, M. A. y Luque, V. A. (2014): "A Vueltas Con Las Sicav. Reflexiones Críticas Desde El Punto De Vista Económico-Tributario" en Revista Papeles de Europa, vol.27, no 2, 2- 17.

Luque, V. A. (2014): "Débil crecimiento y transformación productiva: un análisis de la economía alemana durante el período 1995 - 2007". Disponible en http://eprints.ucm.es/28009/

Malthus, T. R. (1990): "Ensayo sobre el principio de la población”, Akal bolsillo, Madrid.

Martínez-Celorrio, X., \& Marín Saldo, A. (2012): “Educación y movilidad social en España", Informe España 2012. 
Luque, Víctor. A propósito de Piketty: evolución de la desigualdad en España.

Martínez Celorrio, X. (2013): "Tendencias de movilidad y reproducción social por la educación en España”, Revista de la Asociación de Sociología de la Educación (RASE), 2013, vol. 6, num. 1, p. 32-48.

Mian, A., y Sufi, A. (2014): "Piketty and U.S. Wealth Inequality", disponible en http://houseofdebt.org/2014/05/24/piketty-and-u-s-wealth-inequality.html, última vez consultado 1 Junio 2015.

Mian, A., y Sufi, A. (2014): "House of Debt: How They (and You) Caused the Great Recession, and How We Can Prevent It from Happening Again", University of Chicago Press.

Michavila, F. (2013): “La universidad española en cifras 2012", en Conferencia de Rectores de las Universidades Españolas (CRUE).

Ostry, M. J. D., Berg, M. A. y Tsangarides, M. C. G. (2014): "Redistribution, inequality, and growth", International Monetary Fund.

Oxfam, I. (2012): "Crisis, desigualdad y pobreza: aprendizajes desde el mundo en desarrollo ante los recortes sociales en España", Informe de Intermón Oxfam, 32.

Panorama de la educación. Indicadores de la OCDE 2013. Informe español. Instituto Nacional de Evaluación Educativa, Madrid, 2013.

Pagés, J. M., y Maza, L. A. (2003): "Análisis del precio de la vivienda en España", Documentos de trabajo del Banco de España, (7), 7-48.

Persson, T., y Tabellini, G. (1991): "Is inequality harmful for growth? Theory and evidence" (No. w3599). National Bureau of Economic Research.

Pettifor, A., y Tily, G. (2014): "Piketty's determinism?", Real-world economics review, no 69 , pp. 44-50.

Piketty, T. (2014): "Capital in the twenty-first century", Belknap Press, Cambridge, (2013)

Piketty, T. (2015): "Putting Distribution Back at the Center of Economics: Reflections on Capital in the Twenty-First Century". The Journal of Economic Perspectives, 67-88.

Richard C. Koo, (2014): "Piketty's inequality and local versus global Lewis turning points", real-world economics review, issue no. 69, 7 Oct 2014, pp. 89-99.

Syll, L. P. (2014): "Piketty and the limits of marginal productivity theory", Real-world economics review, no 69 , pp. 36-43.

Varoufakis, Y. (2014): "Egalitarianism's latest foe: a critical review of Thomas Piketty's Capital in the Twenty-Frist Century", Real-world economics review, $\mathrm{n}^{\circ}$ 69, pp. 18-35.

Vergés, R. (2012): "Estadísticas de vivienda libre en España. 1987-2011. Series operativas de compraventas y precios", Estadística Española, 54(179), pp. 357-420.

Wade, R. H. (2014): "The Piketty phenomenon and the future of inequality", Realworld economics review, issue no. 69, 7 Oct 2014, 2-17.

Weil, D. N. (2015): "Capital and Wealth in the 21st Century" (No. w20919). National Bureau of Economic Research.

Wilkinson, R. G., Pickett, K. \& Chafer, C. (2011): "The spirit level: Why Greater Equality Makes Societies Stronger", Bloomsbury Publishing USA, Nueva York.

Zucman, G. (2013): "The Missing Wealth of Nations: Are Europe and the US net Debtors or net Creditors?", The Quarterly journal of economics, 128(3), 13211364. 